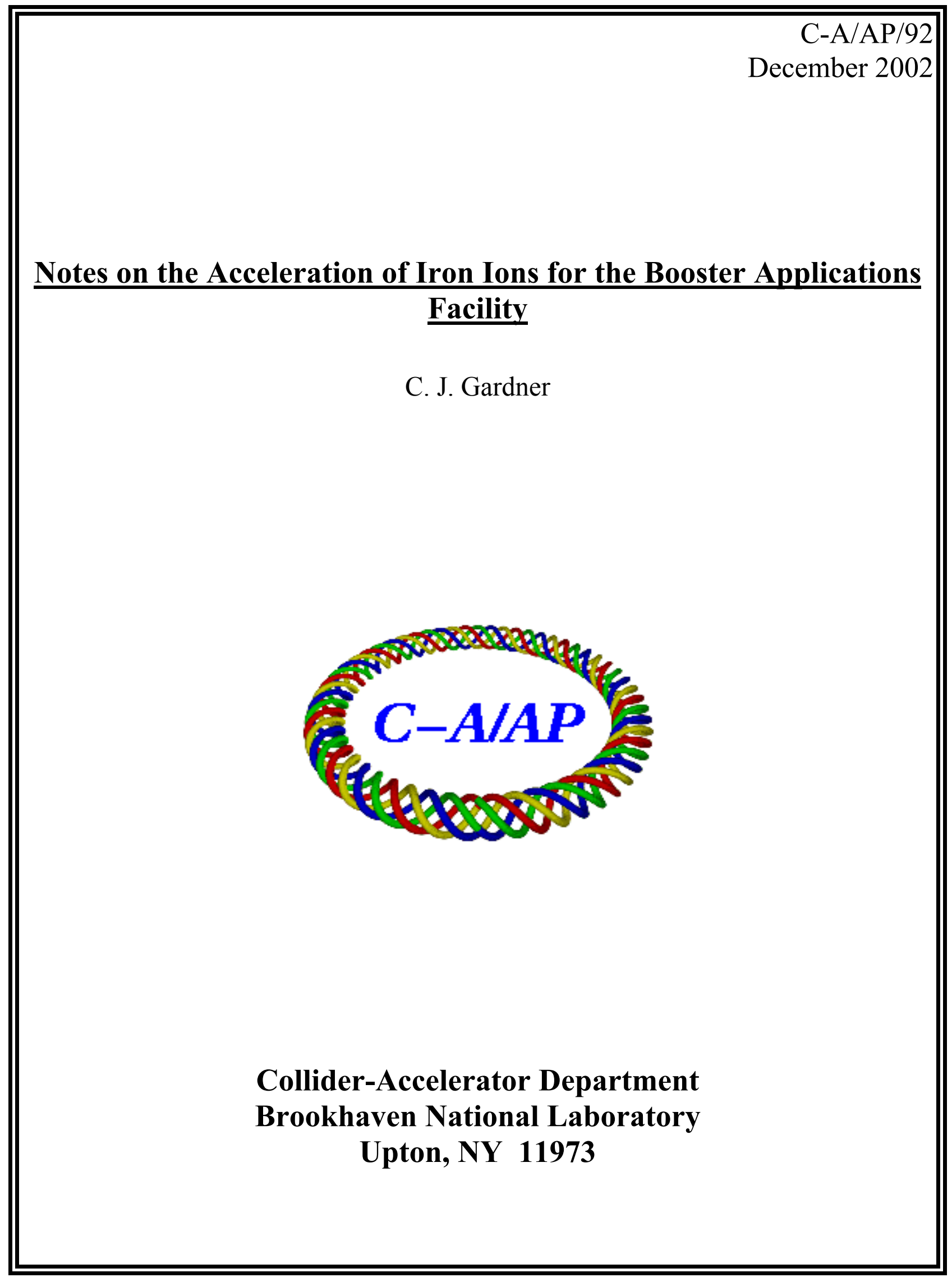




\title{
Notes on the Acceleration of Iron Ions for the Booster Applications Facility
}

\author{
C.J. Gardner
}

December 29, 2002

\section{Introduction}

The Booster Applications Facility (BAF) located near the D and E superperiods of the AGS Booster is a new experimental area designed for the irradiation of material and biological samples with various ions. The ions are extracted from Booster by means of a new resonant extraction system $[1,2]$, and are transported to a target room along a new beamline [3]. The MP7 Tandem Van de Graaff, fed by a pulsed sputter source and connected to Booster by the $840 \mathrm{~m}$ TTB (Tandem To Booster) transport line, provides a wide range of ions for the new facility. The layout of the Tandem, TTB line, and Booster is shown in Figure 1. (The MP6

Tandem, with the indicated bypass line, serves as a spare in the event that MP7 is down for repairs). A closer view of MP7 and the beginning of the TTB line is shown in Figure 2.

Although ions from Tandem have been provided for the irradiation of biological samples for several years now, they have been delivered from the AGS rather than directly from Booster. The ions have been predominantly iron and silicon with kinetic energies of 600 and $1000 \mathrm{MeV}$ per nucleon. Acceleration to these energies has been accomplished by acceleration of $\mathrm{Fe}^{10+}$ and $\mathrm{Si}^{5+}$ in Booster to a kinetic energy of approximately $100 \mathrm{MeV}$ per nucleon and then acceleration of $\mathrm{Fe}^{26+}$ and $\mathrm{Si}^{14+}$ to the final kinetic energy in AGS. The magnetic rigidity in Booster at top energy in this case is approximately $8 \mathrm{Tm}$. In order to reach a kinetic energy of $1000 \mathrm{MeV}$ per nucleon in Booster, iron and silicon ions in charge states higher than $\mathrm{Fe}^{18+}$ and $\mathrm{Si}^{9+}$ are required. The rigidity at top energy is then approximately 16 $\mathrm{Tm}$ which requires a bending field of $1.2 \mathrm{~T}$ in the Booster dipoles. These requirements pose new challenges for Tandem and Booster operation. 
Following are some notes on the operation of Tandem, the TTB line, and Booster for the delivery of iron ions to BAF.

\section{Some Fundamental Parameters}

An iron ion with charge $e Q$ has $N=56$ nucleons, $Z=26$ protons, and $(Z-Q)$ electrons. (Here $Q$ is an integer and $e$ is the charge of a single proton.) The mass and energy are

$$
m=a u-Q m_{e}+E_{b} / c^{2}, \quad E=\sqrt{p^{2} c^{2}+m^{2} c^{4}}
$$

where $a=55.9349421(15)$ is the atomic mass [4] of the neutral iron atom, $u=931.494013 \mathrm{MeV} / c^{2}$ is the unified atomic mass unit [5], $m_{e} c^{2}=.510998902 \mathrm{MeV}$ is the electron mass [5], and $p$ is the momentum. $E_{b}$ is the binding energy of the $Q$ electrons removed from the neutral iron atom. In the calculations that follow, we shall neglect the binding energy. The kinetic energy is

$$
W=E-m c^{2} .
$$

The magnetic rigidity of the ion in units of $\mathrm{Tm}$ is

$$
B \rho=k p / Q
$$

where $k=10^{9} / 299792458$ and $p$ is the momentum in units of $\mathrm{GeV} / \mathrm{c}$. The relativistic parameters $\beta$ and $\gamma$, and the revolution frequency of the ion are

$$
\beta=c p / E, \quad \gamma=E /\left(m c^{2}\right), \quad f=c \beta /(2 \pi R)
$$

where $2 \pi R=201.78 \mathrm{~m}$ is the circumference of the design orbit in Booster. The angular frequency is $\omega=2 \pi f$. We also define the phase-slip factor

$$
\eta=\frac{1}{\gamma_{t}^{2}}-\frac{1}{\gamma^{2}}
$$

where $\gamma_{t}=4.806$ is the transition gamma.

\section{Acceleration in Tandem and TTB Transport}

Negative ions of iron oxide $\left(\mathrm{FeO}^{-}\right)$enter the Tandem with charge $-e$ and mass $m_{s}$, having been accelerated through a potential difference of

$$
V_{s}=130 \mathrm{kV}
$$


in a pulsed sputter source. Neglecting the atomic binding energy, the mass of the iron oxide ion is

$$
m_{s}=a u+b u+m_{e}
$$

where $b=15.9949146221(15)$ is the atomic mass [4] of oxygen.

\subsection{Acceleration to Terminal Foil and Back to Ground}

The part of the $\mathrm{FeO}^{-}$ion consisting of a neutral iron atom, minus $Q_{t}$ electrons, has mass

$$
m_{t}=a u-Q_{t} m_{e}
$$

and gains energy

$$
W_{s}=e V_{s} m_{t} / m_{s}
$$

in the ion source. Upon acceleration from ground potential to the center terminal of Tandem, this part of the ion gains energy

$$
W_{t}=e V_{t} m_{t} / m_{s}
$$

where $V_{t}$ is the terminal voltage. In the center terminal the $\mathrm{FeO}^{-}$ions pass through a thin carbon stripping foil where they separate into iron and oxygen ions in various charge states. Iron ions emerging from the foil with positive charge are accelerated back to ground potential. Those with charge $e Q_{t}$ gain energy $e Q_{t} V_{t}$ and emerge from Tandem with kinetic energy

$$
W_{T}=W_{s}+W_{t}+e Q_{t} V_{t}-\delta W_{t}
$$

where $\delta W_{t}$ is the energy loss in the foil. This equation can be solved to obtain the terminal voltage in terms of $W_{T}$ and $Q_{t}$. One finds

$$
e V_{t}=\frac{W_{T}-W_{s}+\delta W_{t}}{Q_{t}+m_{t} / m_{s}}
$$

\subsection{The Second Stripping Foil}

The terminal voltage is limited to about $14 \mathrm{MV}$ for smooth and reliable operation of Tandem. This means that only iron ions with charge states up to $Q_{t}=+12$ can be produced efficiently in the terminal foil. In order to produce a sufficient number of ions in higher charge states, a second carbon foil is required after acceleration in Tandem. This is located upstream of the first of the two $90^{\circ}$ bends in the TTB line as indicated in 
Figures 1 and 2. Let $Q_{b}$ be the charge state after the second foil. Iron ions with this charge are to be transported down the TTB line and injected into Booster. Acceleration of iron ions to a kinetic energy of $1000 \mathrm{MeV}$ per nucleon in Booster requires $Q_{b} \geq+19$. As of this writing, iron ions with the charge states listed in Table $\mathbf{1}$ have been produced and studied. Here the listed current is the electrical current measured at the TTB beamstop. The number of ions in charge state $Q_{b}$ per source pulse is the electrical current times the pulse width, divided by $Q_{b}$. Taking a pulse width of 300 $\mu$ s we obtain the ions per pulse listed in the table. The surface densities of carbon in the terminal and second foil were 3 and $30 \mu \mathrm{g} / \mathrm{cm}^{2}$ respectively. These were determined to be the optimum foil densities for the production of $\mathrm{Fe}^{20+}$ and $\mathrm{Fe}^{21+}$.

Table 1: Iron Charge States and Intensities

\begin{tabular}{|c|c|c|c|}
\hline$Q_{t}$ & $Q_{b}$ & Current & Ions/Pulse \\
\hline+10 & +20 & $40 \mu \mathrm{A}$ & $3.7 \times 10^{9}$ \\
+11 & +21 & $20 \mu \mathrm{A}$ & $1.8 \times 10^{9}$ \\
\hline
\end{tabular}

\subsection{Momentum Selection}

The charge and momentum of ions emerging from the second foil is selected by the first of the two $90^{\circ}$ bends in the TTB line. A pair of slits (one on either side of the beam) located between the two bends, as indicated in Figures 1 and 2, serves to define the path that corresponds to the desired momentum. Each slit intercepts a small portion of the beam passing through; this provides electrical feedback to keep the terminal voltage at the value required to give the desired momentum. The field in the bends is monitored with NMR probes and is set to give the magnetic rigidity corresponding to the desired momentum. The advertised radius of curvature of the nominal trajectory in the bends is $1.52683 \mathrm{~m}$.

\subsection{Terminal Voltage and Ion Parameters in the TTB Line}

If $e Q_{b}$ is the ion charge after the second foil, then the mass of the ion to be transported to Booster is

$$
m_{b}=a u-Q_{b} m_{e}
$$


It is convenient to parameterize the terminal voltage and other ion parameters in terms of the kinetic energy after the second foil

$$
W=\left(m_{b} / m_{t}\right) W_{T}-\delta W_{f}
$$

where $W_{T}$ is given by (11) and $\delta W_{f}$ is the energy loss in the (second) foil. The momentum is

$$
c p=\sqrt{W^{2}+2 m_{b} c^{2} W} .
$$

Solving (14) for $W_{T}$ and using the result in (12) we have

$$
e V_{t}=e V_{t}^{0}+e \delta V_{t}
$$

where

$$
e V_{t}^{0}=\frac{\left(m_{t} / m_{b}\right) W-W_{s}}{Q_{t}+m_{t} / m_{s}} \approx \frac{W-W_{s}}{Q_{t}+m_{t} / m_{s}}
$$

and

$$
e \delta V_{t}=\frac{\left(m_{t} / m_{b}\right) \delta W_{f}+\delta W_{t}}{Q_{t}+m_{t} / m_{s}} \approx \frac{\delta W_{f}+\delta W_{t}}{Q_{t}+m_{t} / m_{s}} .
$$

Putting $Q_{t}=10$ and $Q_{b}=20$, and using equations (3-4), (7-9), (13), (15) and (17), we obtain the terminal voltages and ion parameters listed in

Table 2 for various values of $W$. Putting $Q_{t}=11$ and $Q_{b}=21$, we obtain the parameters listed in Table $\mathbf{3}$.

Table 2: Parameters obtained with $Q_{t}=10$ and $Q_{b}=20$

\begin{tabular}{|c|c|c|c|c|}
\hline$W(\mathrm{MeV})$ & $V_{t}^{0}(\mathrm{MV})$ & $c p(\mathrm{MeV} / n)$ & $B \rho(\mathrm{Tm})$ & $\beta$ \\
\hline 152.611146 & 14.152 & 71.25691 & 0.665525 & 0.07637768 \\
153.611146 & 14.245 & 71.49033 & 0.667705 & 0.07662641 \\
154.611146 & 14.338 & 71.72299 & 0.669878 & 0.07687432 \\
\hline
\end{tabular}

Table 3: Parameters obtained with $Q_{t}=11$ and $Q_{b}=21$

\begin{tabular}{|c|c|c|c|c|}
\hline$W(\mathrm{MeV})$ & $V_{t}^{0}(\mathrm{MV})$ & $c p(\mathrm{MeV} / n)$ & $B \rho(\mathrm{Tm})$ & $\beta$ \\
\hline 168.332433 & 14.285 & 74.84251 & 0.665727 & 0.08019761 \\
169.332433 & 14.370 & 75.06485 & 0.667705 & 0.08043432 \\
170.332433 & 14.455 & 75.28653 & 0.669677 & 0.08067031 \\
\hline
\end{tabular}

Here the notation "MeV/ $n$ " for the unit of $c p$ means "MeV per nucleon". The nominal kinetic energy of the $\mathrm{Fe}^{20+}$ and $\mathrm{Fe}^{21+}$ ions to be transported 
to Booster are 153.611146 and $169.332433 \mathrm{MeV}$ respectively. The corresponding momenta are 71.49033 and $75.06485 \mathrm{MeV}$ per nucleon. The masses of the ions are 52.0928437 and $52.0923327 \mathrm{GeV} / c^{2}$ respectively. Note that the listed magnetic rigidities are some $20 \%$ lower than the nominal rigidity, $B \rho=0.854085$, for gold $\left(\mathrm{Au}^{32+}\right)$ ions transported to Booster during RHIC operation. This means that the iron ions are somewhat more susceptible to magnetic field errors in the TTB line and in Booster at injection. Table 4 summarizes the nominal parameter values for $\mathrm{Fe}^{20+}$ and $\mathrm{Fe}^{21+}$ along with those for $\mathrm{Fe}^{10+}$.

Table 4: Nominal Parameters

\begin{tabular}{|c|c|c|c|c|c|c|}
\hline$Q_{t}$ & $Q_{b}$ & $W(\mathrm{MeV})$ & $V_{t}^{0}(\mathrm{MV})$ & $c p(\mathrm{MeV} / n)$ & $B \rho(\mathrm{Tm})$ & $\beta$ \\
\hline 10 & 10 & 127.686665 & 11.838 & 65.17425 & 1.21742815 & 0.06988440 \\
\hline 10 & 20 & 153.611146 & 14.245 & 71.49033 & 0.667705 & 0.07662641 \\
\hline 11 & 21 & 169.332433 & 14.370 & 75.06485 & 0.667705 & 0.08043432 \\
\hline
\end{tabular}

Note that these values have been obtained in the following way. The measured revolution frequency of $\mathrm{Fe}^{10+}$ ions at injection in Booster is $622.98 / 6 \mathrm{kHz}$. Assuming an orbit circumference of $201.78 \mathrm{~m}$ then gives a magnetic rigidity of $1.21742815 \mathrm{Tm}$. The corresponding measured field in the $90^{\circ}$ bends of the TTB line is 7958.15 gauss. For the transport of $\mathrm{Fe}^{20+}$, the measured field in the $90^{\circ}$ bends is 4364.69 gauss. The nominal rigidity for $\mathrm{Fe}^{20+}$ is then $1.21742815 \times(4364.69 / 7958.15)=0.66770499 \mathrm{Tm}$. The nominal rigidity for $\mathrm{Fe}^{21+}$ is taken to be the same as that for $\mathrm{Fe}^{20+}$.

\subsection{Transport to Booster}

Downstream of the $90^{\circ}$ bends, the TTB line contains two $24^{\circ}$ and two $13^{\circ}$ bends. (Each pair is depicted as just one bend in Figure 1). Quadrupoles between the bends of each pair are adjusted to make the pair achromatic. Focusing in the line is accomplished with a series of quadrupole doublets. A beamstop is located downstream of the second $13^{\circ}$ bend. The transport efficiency of the line depends on the horizontal and vertical emittances of the iron beam to be transported. If the $\mathrm{Fe}^{20+}$ and $\mathrm{Fe}^{21+}$ emittances are 
comparable to those of $\mathrm{Au}^{32+}$, then the efficiency is expected to be 85 to 95\%. (The $95 \%$ emittances of the $\mathrm{Au}^{32+}$ beam are estimated to be of the order of $1 \pi \mathrm{mm}$ milliradian, unnormalized). As of this writing, beam currents of $35-40 \mu \mathrm{A}$ of $\mathrm{Fe}^{20+}$ have been observed at the TTB beamstop; $15-20 \mu \mathrm{A}$ of $\mathrm{Fe}^{21+}$ have been observed at the Booster inflector.

\section{Booster Injection}

\subsection{Injection Parameters}

Iron ions from the TTB line are injected into Booster by means of an electrostatic inflector [6] and four programmable injection kickers as depicted schematically in Figure 3. The kickers produce a horizontal closed orbit bump which allows beam emerging from the inflector to be placed within the Booster acceptance. Tables 5 and $\mathbf{6}$ list the $\mathrm{Fe}^{20+}$ and $\mathrm{Fe}^{21+}$ injection parameters for various values of the kinetic energy $W$. Here $V_{I}$ is the voltage required between the cathode and septum of the inflector, $f$ is the revolution frequency, and $h$ is the RF harmonic. The inflector voltage $V_{I}$ is given by

$$
e V_{I}=\frac{G}{R_{I}} c^{2} p^{2} /\left(Q_{b} E\right)
$$

where $G=0.017 \mathrm{~m}$ is the gap between the cathode and septum, and $R_{I}=8.74123 \mathrm{~m}$ is the radius-of-curvature along the nominal trajectory through the inflector. The values of $h f$ are calculated assuming RF

harmonic $h=3$. The nominal injection parameters for $\mathrm{Fe}^{20+}$ and $\mathrm{Fe}^{21+}$ are those corresponding to kinetic energies $W=153.611146$ and 169.332433 $\mathrm{MeV}$ respectively. These are listed in Table 7 along with the nominal values for $\mathrm{Fe}^{10+}$.

Table 5: $\mathrm{Fe}^{20+}$ Injection Parameters

\begin{tabular}{|c|c|c|c|c|}
\hline$W(\mathrm{MeV})$ & $V_{I}(\mathrm{kV})$ & $c p(\mathrm{MeV} / n)$ & $B \rho(\mathrm{Tm})$ & $h f(\mathrm{kHz})$ \\
\hline 152.611146 & 29.637 & 71.25691 & 0.665525 & 340.432 \\
153.611146 & 29.830 & 71.49033 & 0.667705 & 341.541 \\
154.611146 & 30.024 & 71.72299 & 0.669878 & 342.646 \\
\hline
\end{tabular}


Table 6: $\mathrm{Fe}^{21+}$ Injection Parameters

\begin{tabular}{|c|c|c|c|c|}
\hline$W(\mathrm{MeV})$ & $V_{I}(\mathrm{kV})$ & $c p(\mathrm{MeV} / n)$ & $B \rho(\mathrm{Tm})$ & $h f(\mathrm{kHz})$ \\
\hline 168.332433 & 31.128 & 74.84251 & 0.665727 & 357.458 \\
169.332433 & 31.313 & 75.06485 & 0.667705 & 358.513 \\
170.332433 & 31.498 & 75.28653 & 0.669677 & 359.565 \\
\hline
\end{tabular}

Table 7: Nominal Injection Parameters

\begin{tabular}{|c|c|c|c|c|c|c|c|}
\hline$Q_{t}$ & $Q_{b}$ & $W(\mathrm{MeV})$ & $V_{I}(\mathrm{MV})$ & $c p(\mathrm{MeV} / n)$ & $B \rho(\mathrm{Tm})$ & $h$ & $h f(\mathrm{kHz})$ \\
\hline 10 & 10 & 127.686665 & 49.604 & 65.17425 & 1.21742815 & 6 & 622.98 \\
\hline 10 & 20 & 153.611146 & 29.830 & 71.49033 & 0.66770499 & 3 & 341.541 \\
\hline 11 & 21 & 169.332433 & 31.313 & 75.06485 & 0.66770499 & 3 & 358.513 \\
\hline
\end{tabular}

\subsection{Dipole Magnetic Field at Injection}

The magnetic field required in the bending dipoles of Booster at injection is given by

$$
B=(B \rho) / \rho
$$

where $\rho=13.8656$ meters is the nominal radius of curvature in the dipoles and $B \rho$ has the values listed in Table 7 . (There are 36 bending dipoles in the Booster lattice; each has a magnetic length of $l=2.42$ meters and bends the beam by $\theta=2 \pi / 36=\pi / 18$ radians. The nominal radius of curvature is then $\rho=l / \theta=2.42 \times 18 / \pi$.) The nominal magnetic field at injection is 481.55506 gauss for $\mathrm{Fe}^{20+}$ and $\mathrm{Fe}^{21+}$. This is some $20 \%$ lower than the nominal 615.97436 gauss for $\mathrm{Au}^{32+}$.

\subsection{Multi-turn Injection}

Since the revolution periods of the $\mathrm{Fe}^{20+}$ and $\mathrm{Fe}^{21+}$ ions in Booster are 8.78 and $8.37 \mu$ s respectively, injection of a $300 \mu$ s pulse from Tandem occurs over a period of some 35 turns around the machine. The closed orbit bump produced by the kickers initially places the orbit near the 
septum at the exit of the inflector. As beam is injected and begins to circulate, the bump must be collapsed at a rate sufficient to keep the circulating beam from hitting the inflector. The incoming beam is thereby deposited into a series of phase space layers surrounding the orbit. The collapse continues until the orbit is so far from the septum that any additional incoming beam will be injected outside the $185 \pi$ ( $\mathrm{mm}$ milliradians) horizontal acceptance of the machine. This is a delicate process that requires careful tuning to achieve the highest injection efficiency. Experience with $\mathrm{Au}^{32+}$ ions $[7,8,9]$ has shown that with the introduction of linear coupling, 35 turns can be injected with up to $85 \%$

efficiency. One expects similar efficiency for $\mathrm{Fe}^{20+}$ and $\mathrm{Fe}^{21+}$ provided the tansverse emittance is comparable to that of $\mathrm{Au}^{32+}$.

\section{$5 \quad$ RF Capture and Acceleration}

\subsection{Harmonic Number}

Capture and acceleration of the injected beam is accomplished with two RF cavities (A3 and B3). These can operate in the range of roughly 400 $\mathrm{kHz}$ to $5 \mathrm{MHz}$. For the acceleration of $\mathrm{Au}^{32+}$ ions to a kinetic energy of $101 \mathrm{MeV}$ per nucleon for RHIC operation, harmonic $h=6$ is used and the cavity frequency $h f$ goes from $398 \mathrm{kHz}$ at injection to $3.85 \mathrm{MHz}$ at top energy. For $\mathrm{Fe}^{20+}$ and $\mathrm{Fe}^{21+}$ ions with a kinetic energy of $1000 \mathrm{MeV}$ per nucleon, the frequency $h f$ would be $7.81 \mathrm{MHz}$ at harmonic $h=6$, which is well above the tuning range of the cavities. At harmonic $h=4$, one has $h f=455$ and $478 \mathrm{kHz}$ at injection for $\mathrm{Fe}^{20+}$ and $\mathrm{Fe}^{21+}$ respectively, and $h f=5.21 \mathrm{MHz}$ at top energy. Here the frequencies at injection are certainly within the range of the cavities, but the frequency at top energy may be too high. At harmonic $h=3$ one has $h f=342$ and $359 \mathrm{kHz}$ at injection, and $h f=3.91 \mathrm{MHz}$ at top energy. Here the frequencies at injection may be too low. The various frequencies are summarized in Table 8 .

If the cavities can be made to operate in the range of $450 \mathrm{kHz}$ to $5.2 \mathrm{MHz}$, or in the range of $340 \mathrm{kHz}$ to $3.9 \mathrm{MHz}$, then the entire acceleration to 1000 $\mathrm{MeV}$ per nucleon could be carried out at one harmonic. Otherwise, one possible scenario would be to capture at harmonic $h=6$, accelerate a bit, merge the six bunches into three, and then continue acceleration to full energy at harmonic $h=3$. As of this writing, $\mathrm{Fe}^{21+}$ has been accelerated 
Table 8: Harmonic Number and Cavity Frequencies

\begin{tabular}{|c|c|c|c|c|}
\hline Ion & $h$ & $h f$ Injection & $h f$ Extraction & $W$ Extraction \\
\hline \multirow{2}{*}{$\mathrm{Au}^{32+}$} & 6 & $398 \mathrm{kHz}$ & $3.85 \mathrm{MHz}$ & $101 \mathrm{MeV} / n$ \\
\hline \multirow{2}{*}{$\mathrm{Fe}^{20+}$} & 6 & $683 \mathrm{kHz}$ & $7.81 \mathrm{MHz}$ & \\
& 4 & $455 \mathrm{kHz}$ & $5.21 \mathrm{MHz}$ & $1000 \mathrm{MeV} / n$ \\
& 3 & $342 \mathrm{kHz}$ & $3.91 \mathrm{MHz}$ & \\
$\mathrm{Fe}^{21+}$ & 6 & $717 \mathrm{kHz}$ & $7.81 \mathrm{MHz}$ & \\
& 4 & $478 \mathrm{kHz}$ & $5.21 \mathrm{MHz}$ & $1000 \mathrm{MeV} / n$ \\
& 3 & $359 \mathrm{kHz}$ & $3.91 \mathrm{MHz}$ & \\
\hline
\end{tabular}

at harmonic $h=4$ in Booster to a kinetic energy of $851 \mathrm{MeV}$ per nucleon. The measured frequency at this energy was $h f=5.0686 \mathrm{MHz}$. Mike Brennan believes that capture and acceleration to $1000 \mathrm{MeV}$ per nucleon can be carried out at harmonic $h=3$.

\subsection{Adiabatic Capture and Electron Capture Losses}

During injection and capture, the Booster magnetic field is held constant and beam is captured into stationary RF buckets with the net RF voltage raised adiabatically from zero. (The two cavities are initially counterphased so that the net voltage seen by the beam is zero. By gradually decreasing the amount of counterphasing, the net voltage can be raised slowly). For the capture of $\mathrm{Au}^{32+}$ ions we have found $[7,8]$ that extending the time allowed for adiabatic capture on the Booster injection porch from the 1-3 ms used in the past to approximately $6 \mathrm{~ms}$ improves beam survival. This is contrary to the expectation that spending more time at low energy produces more beam loss. Here the cross sections for electron capture interactions between gold and residual gas or ions in the vacuum chamber are relatively large [10]. Clearly, if too much time is spent at low energy, these interactions will produce significant loss. On the other hand, if too little time is spent on capture, there can be substantial capture loss. The loss itself will generate more residual gas or ions in the vacuum chamber thereby increasing the rate of loss due to electron capture. One therefore expects some sort of optimum setup in which the benefits of reducing capture loss outweigh the cost of spending more time at low energy. The optimum setup for iron ions has yet to be determined. 


\subsection{Acceleration}

After capture, the magnetic field is ramped and beam is accelerated to top energy. The required RF voltage is given by

$$
V_{R F} \sin \phi_{s}=2 \pi R \rho \dot{B} / c
$$

where $\phi_{s}$ is the synchronous phase, $R$ is the radius, $\rho$ is the radius of curvature, and Bdot $(\dot{B})$ is the time derivative of $B$. Because the BMM (Booster Main Magnet) power supply cannot operate at full voltage at the top energy of $1000 \mathrm{MeV}$ per nucleon, Bdot is limited to approximately 3 $\mathrm{T} / \mathrm{s}$ throughout the magnetic cycle. (This is approximately one third of the maximum Bdot reached during the acceleration of $\mathrm{Au}^{32+}$ to $101 \mathrm{MeV}$ per nucleon.) An analysis similar to that carried out in [11] for $\mathrm{Au}^{32+}$ shows that the available $\mathrm{RF}$ voltage $(30 \mathrm{kV})$ is more than adequate for the acceleration of $\mathrm{Fe}^{20+}$ and $\mathrm{Fe}^{21+}$.

The momentum, rigidity, beta, and frequency for $\mathrm{Fe}^{20+}$ and $\mathrm{Fe}^{21+}$ at various top energies $(W)$ are listed in Tables $\mathbf{9}$ and $\mathbf{1 0 .}$

Table 9: $\mathrm{Fe}^{20+}$ Parameters at Top Energy

\begin{tabular}{|c|c|c|c|c|}
\hline$B \rho(\mathrm{Tm})$ & $W(\mathrm{MeV} / n)$ & $c p(\mathrm{MeV} / n)$ & $\beta$ & $h f(\mathrm{MHz})$ \\
\hline 15.696293 & 990.6255 & 1680.5822 & 0.87491371 & 3.899681 \\
15.796293 & 1000.0000 & 1691.2891 & 0.87621146 & 3.905465 \\
15.896293 & 1009.3884 & 1701.9960 & 0.87749042 & 3.911166 \\
\hline
\end{tabular}

Table 10: $\mathrm{Fe}^{21+}$ Parameters at Top Energy

\begin{tabular}{|c|c|c|c|c|}
\hline$B \rho(\mathrm{Tm})$ & $W(\mathrm{MeV} / n)$ & $c p(\mathrm{MeV} / n)$ & $\beta$ & $h f(\mathrm{kHz})$ \\
\hline 14.944040 & 990.1570 & 1680.0414 & 0.87484967 & 3.899395 \\
15.044040 & 1000.0000 & 1691.2836 & 0.87621280 & 3.905471 \\
15.144040 & 1009.8581 & 1702.5259 & 0.87755521 & 3.911455 \\
\hline
\end{tabular}

Here the values of $h f$ are calculated assuming RF harmonic $h=3$. The nominal top energy is $1000 \mathrm{MeV}$ per nucleon.

Assuming the beam fills the horizontal and vertical acceptances at injection, one expects normalized horizontal and vertical emittances of $14 \pi$ 
and $6.7 \pi$ (mm milliradians) respectively throughout the acceleration cycle. For $\mathrm{Au}^{32+}$ ions, the combined capture and acceleration efficiency is about $80 \%$. As of this writing, approximately $8 \times 10^{8} \mathrm{Fe}^{21+}$ ions have been captured with $4 \times 10^{8}$ of these accelerated to $851 \mathrm{MeV}$ per nucleon.

\section{The Magnetic Cycle}

\subsection{Field and Current at Injection and Top Energy}

The magnetic field produced by the 16-turn main winding on the Booster dipole is given by [12]

$$
B=K I
$$

where $K=2.43$ gauss/A and $I$ is the current in the winding. Using (20) and the magnetic rigidities listed in Tables 5, 6, 9 and 10 one obtains the fields and currents listed in Table $\mathbf{1 1}$ for gold and iron ions at injection and at top energy. Here we see that for the acceleration of $\mathrm{Fe}^{20+}$, the field

Table 11: Booster Dipole Fields and Currents

\begin{tabular}{|c|c|c|c|c|}
\hline Ion & $W(\mathrm{MeV} / n)$ & $B \rho(\mathrm{Tm})$ & $B$ (gauss) & $I$ (Amps) \\
\hline & $182.8790 / 197$ & 0.854085 & 616 & 253 \\
$\mathrm{Au}^{32+}$ & 101.1721 & 9.152950 & 6601 & 2716 \\
\hline \multirow{3}{*}{$\mathrm{Fe}^{20+}$} & $153.6111 / 56$ & 0.667705 & 482 & 198 \\
& 1000 & 15.796293 & 11392 & 4688 \\
$\mathrm{Fe}^{21+}$ & $169.3324 / 56$ & 0.667705 & 482 & 198 \\
& 1000 & 15.044040 & 10850 & 4465 \\
\hline
\end{tabular}

goes from 482 gauss at injection to 11392 gauss at top energy. The corresponding current goes from 198 to 4688 A. As of this writing, Fe ${ }^{21+}$ has been accelerated to a top field of approximately 9750 gauss; the corresponding current was $4012 \mathrm{~A}$.

\subsection{Voltage Limitations at High Field}

The current is provided by the BMM power supply which consists of six modules connected in series. Each module provides a maximum of $1000 \mathrm{~V}$, but only two of the six can operate at the nearly 5000 A required for the 
acceleration of $\mathrm{Fe}^{20+}$ and $\mathrm{Fe}^{21+}$ to $1000 \mathrm{MeV}$ per nucleon. This means that for any magnetic cycle requiring high current, the four lower-current modules must be bypassed. The available voltage for such cycles is therefore reduced from 6000 to $2000 \mathrm{~V}$. As already noted, the maximum Bdot in this case is limited to approximately $3 \mathrm{~T} / \mathrm{s}$. In order to keep the ripple during extraction to a minimum, one needs to bypass one of the high-current modules during the flat top portion of the magnetic cycle. Originally this could be done only by bypassing the module for the entire cycle. This left just one module to be used for the entire cycle with the available voltage limited to $1000 \mathrm{~V}$. In this case the maximum Bdot is limited to approximately $1.5 \mathrm{~T} / \mathrm{s}$. Software now has been developed so that the needed high-current module can be switched on during the acceleration and ramp-down portions of the magnetic cycle and bypassed during flat top.

\subsection{Magnetic Cycles for Slow Extraction}

At top energy the beam is to be extracted over a period of several hundred to $1000 \mathrm{~ms}$. Figure 4 shows a possible magnetic cycle for the acceleration and extraction of $\mathrm{Fe}^{20+}$. Here the flattop at $1000 \mathrm{MeV}$ per nucleon (11.4 $\mathrm{kG}$ ) is $700 \mathrm{~ms}$ long and the cycle length is 4 seconds. The voltage is kept between \pm 900 volts so that only one of the high-current modules is required. Note that although the cycle length here is 4 seconds, an additional 2 seconds must be added to the dwell field time in order to keep the rms current sufficiently low. This gives a total cycle time of 6 seconds. The settings of the magnetic field $B$ used to generate the cycle are listed in Table 12. Here $t$ is the time during the cycle and the column labeled "Interpolation" lists the type of iterpolation between a given point and the preceding point. The final column lists the slope $m$ of the function $B(t)$.

Figure 5 shows a magnetic cycle in which both of the high-current modules are used during acceleration and the down-ramp. Here the flattop at $1000 \mathrm{MeV}$ per nucleon $(11.4 \mathrm{kG})$ is again $700 \mathrm{~ms}$ long but the cycle length has been reduced to 2 seconds. The voltage is kept between \pm 1800 volts. The settings of the magnetic field for the cycle are listed in Table 13. 


\section{Tunes during the Magnetic Cycle}

The horizontal and vertical tunes, $Q_{H}$ and $Q_{V}$, are controlled by excitation of the one-turn pancake windings located on each of the 48 ring quadrupoles. The windings on the 24 quads located at horizontal beta maximums are connected together in one series string as are those on the 24 quads located at vertical beta maximums. The two strings are called the horizontal and vertical tune quads respectively and are powered by two \pm 1100 A programmable power supplies. (The five-turn main windings on the quadrupoles are connected in series with the main windings on the ring dipoles and are powered by the BMM power supply.)

\subsection{The Effect of Quadrupole Iron Saturation}

At high field, the Booster quadrupole iron begins to saturate before the dipole iron, which weakens the strength of the quadrupole relative to the dipole and lowers the machine tunes. Measurements reported in $[13,14]$ show that the quadrupoles begin to saturate when the current in the main windings reaches $2500 \mathrm{~A}$. (The field in the dipoles at this current is 0.6075 $\mathrm{T}$ and the magnetic rigidity is $8.42 \mathrm{Tm}$.) Using the measured quadrupole strengths, the tunes for various quadrupole currents have been calculated with the MAD program [15] and the results have been incorporated into the Booster Tune Control program (also called Optics Control). This program is used to manipulate the tunes throughout the magnetic cycle and is described in [16]. Gardner [17] has used the Tune Control program to calculate the regions of Booster tune space that can be accessed at magnetic rigidities of $12.5,14.1$ and 16.0 Tm assuming currents of $\pm 700 \mathrm{~A}$ in the quadrupole strings. More recently, a high precision MAD model of the Booster tunes has been developed by Brown [18]. Figure 6 shows the regions of tune space that are accessible according to this model. Here the quadrupole strings are excited with currents of $\pm 1000 \mathrm{~A}$ and the resulting borders of the regions that are accessible for rigidities of 14, 15, 16 and 17 Tm are plotted. One sees that the area of the upper-right tune space quadrant

$$
4.5<Q_{H}<5.0, \quad 4.5<Q_{V}<5.0
$$

that can be accessed shrinks as the rigidity increases beyond $14 \mathrm{Tm}$. The bare tunes, obtained with zero current in the quad strings, are also plotted in the figure. These move toward the lower-left quadrant as the rigidity increases. At a rigidity of $17 \mathrm{Tm}$, only a very small portion of the 
upper-right quadrant can be accessed. The Booster's nominal operating point

$$
Q_{H}=4.82, \quad Q_{V}=4.83
$$

therefore cannot be reached at high rigidity, and, in particular, one cannot reach the $Q_{H}=4+2 / 3$ line for resonant extraction. One is forced to move $Q_{H}$ below 4.5 at high field so that the $Q_{H}=4+1 / 3$ resonance can be used. In order to reach this line, the original $\pm 700 \mathrm{~A}$ power supplies for the two strings have been replaced with new \pm 1100 A supplies. The cables in the tune quadrupole strings have been upgraded to accommodate the higher current.

\subsection{Tunes at Injection}

For the multiturn injection of $\mathrm{Fe}^{20+}$ and $\mathrm{Fe}^{21+}$, one can use the "standard" coupled injection setup $[7,8,9]$ used for the injection of gold ions in Booster. The nominal uncoupled tunes for this setup are near the point $Q_{H}=4.757$ and $Q_{V}=4.777$ which is indicated by the green dot in Figure 7. Subsequent extraction with $Q_{H}=4+1 / 3$ requires that several resonance lines (including $Q_{H}=4.5$ ) be crossed. Experience with $\mathrm{Au}^{32+}$ beam has shown that the loss upon crossing the various lines can be eliminated by resonance correction and by crossing with sufficient speed. This must be done at low energy shortly after injection where the resonance corrections have sufficient strength. The tunes are moved along the path indicated by the dashed line in the figure, first to the blue point, which has tunes $Q_{H}=4.4$ and $Q_{V}=4.74$, and then to the black point, which has tunes $Q_{H}=4.38$ and $Q_{V}=4.55$.

Another option would be to inject $\mathrm{Fe}^{20+}$ and $\mathrm{Fe}^{21+}$ with $Q_{H}$ and $Q_{V}$ below 4.5. This would eliminate the need to cross the resonances, but would require a new coupled injection setup. As with the standard setup, the uncoupled tunes need to be near the $Q_{H}=Q_{V}$ resonance line. One possible choice is

$$
Q_{H}=Q_{0}-0.01, \quad Q_{V}=Q_{0}+0.01
$$

where

$$
Q_{0}=4+13 / 30=4.433333 .
$$

These tunes are indicated by the red dot in Figure 7. The current in the skew quads would then be adjusted to give the normal-mode tunes

$$
Q_{1}=4+12 / 30=4+6 / 15=4.400000
$$


and

$$
Q_{2}=4+14 / 30=4+7 / 15=4.466667 .
$$

As the injected beam is accumulated, the coupling strength needs to be reduced so that the vertical emittance of the accumulated beam does not exceed the $87 \pi$ vertical acceptance of the ring. This can be done by increasing the uncoupled tune separation as the beam is accumulated. In this case the uncoupled tunes would be moved to

$$
Q_{H}=4.38, \quad Q_{V}=4.55
$$

which is indicated by the black dot in Figure 7. Modeling of this particular setup shows that injection efficiencies comparable to those of the standard setup can be achieved. The solid black line connecting the red and black dots shows that the $Q_{V}=4.5$ resonance must be crossed. In principle this can be done with no beam loss by applying the necessary resonance correction and by crossing with sufficient speed.

\subsection{Tunes during Acceleration and Extraction}

During acceleration and prior to extraction, the tunes are held at the point $Q_{H}=4.38$ and $Q_{V}=4.55$ (indicated by the black dot in Figure 7) which is near the center of the region bounded by the resonance lines $Q_{V}=4.5$, $3 Q_{H}=13$, and $Q_{H}+Q_{V}=9$. The tune spreads must be kept small enough to avoid loss on these lines. (Note that according to J.W. Glenn, the horizontal and vertical tune spreads just prior to resonant extraction in AGS are approximately 0.2 and 0.02 respectively.)

After acceleration, the slow extraction process is initiated by moving $Q_{H}$ toward the $3 Q_{H}=13$ resonance.

\section{Extraction}

Iron ions for BAF are extracted from Booster by a new resonant extraction system as detailed in $[1,2]$. The system consists of two septum magnets, five bump magnets, and four drive sextupoles.

\subsection{Septum Magnets}

The septum magnets are dipoles located in the D3 and D6 straight sections. The "thin" septum of the D3 magnet (effectively $0.76 \mathrm{~mm}$ thick) 
is the first crossed by the resonant beam. Having crossed this septum, the iron ions then cross the "thick" septum of the D6 magnet (effectively 15.2 $\mathrm{mm}$ thick) and are extracted from the machine. Just upstream of the D6 magnet, ions that have crossed the D3 septum pass through a foil where the remaining electrons are stripped away. This reduces the ion rigidity thereby reducing the magnetic field required in the D6 magnet and in the BAF transport line.

\subsection{Bump Magnets}

The Booster dipole has a main winding consisting of 16 turns, a flat trim winding consisting of 2 turns, and an additional low-current trim winding consisting of 10 turns. The magnetic fields produced by the main and flat trim windings are respectively

$$
B=K I, \quad \Delta B=(K / 8) \Delta I
$$

where $I$ and $\Delta I$ are the corresponding currents and $K=2.43$ Gauss/A as reported by Thern [12]. Local bumps in the equilibrium orbit near the D3 and D6 septa are produced by exciting the flat trim windings on dipole magnets C7, D1, D4, D7 and E1. Each of these windings is connected to its own \pm 600 A programmable power supply for this purpose. (The available voltage from each supply is $\pm 40 \mathrm{~V}$ ). The wiring diagram for the five bump magnets is shown in Figure 8. The indicated polarities follow the conventions of Ref. [19].

During proton operation, the flat trim winding on the D1 dipole is used along with the windings on dipoles $\mathrm{C} 4$ and $\mathrm{C} 8$ to produce the so-called Slow Injection Bump at the H-minus injection foil. The D1 winding is connected to a $\pm 50 \mathrm{~A}$ power supply for this purpose. For BAF operation, the $\pm 50 \mathrm{~A}$ supply is disconnected and the $\pm 600 \mathrm{~A}$ supply is connected in its place. One way to avoid having to switch power supplies when going back and forth between proton and BAF operation would be to use the \pm 600 A supply for both modes of operation. This will work provided the \pm 600 A supply can regulate sufficiently well at the low currents required for the Slow Injection Bump. Another option would be to connect the \pm 50 A supply across the \pm 600 A supply. Yet another option would be to connect the $\pm 50 \mathrm{~A}$ supply to two turns of the low-current winding on the dipole. This will work provided the low-current turns can carry $50 \mathrm{~A}$. (Eight of the ten low-current turns are connected to the eddy current correction winding on the vacuum chamber. The remaining two are used 
as a monitor winding, but presumably could be taken out of the monitor system and used for the slow injection bump.)

\subsection{Sextupoles}

Each superperiod of Booster contains eight sextupoles which are labeled SVX1, SHX2, SVX3, SHX4, SVX5, SHX6, SVX7, and SHX8, where SH and SV denote, respectively, sextupoles located near horizontal and vertical beta maximums, and $\mathrm{X}$ refers to superperiod $\mathrm{A}, \mathrm{B}, \mathrm{C}, \mathrm{D}, \mathrm{E}$, or F. We shall refer to the $\mathrm{SH}$ and SV sextupoles as horizontal and vertical sextupoles respectively. The sextupoles have a main winding consisting of 8 turns, a monitor winding consisting of one turn, and an auxiliary winding consisting of either one or two turns. The measured integrated strength of the windings is $B^{\prime \prime} L=1.643 \times 10^{-3} \mathrm{~T} / \mathrm{m}$ per Ampere-turn [20]. (Here $B$ is the vertical magnetic field on the midplane of the sextupole, $L$ is the magnetic length, and the primes denote differentiation with respect to the horizontal coordinate.)

\subsubsection{Chromaticity and Drive Sextupole Strings}

The main windings are connected together to form four series strings called the Horizontal and Vertical strings and the $\mathrm{C}$ and $\mathrm{F}$ strings. The Horizontal and Vertical strings are used for chromaticity adjustment; the $\mathrm{C}$ and $\mathrm{F}$ strings are used for excitation of the $3 Q_{H}=13$ resonance. Within each string the sextupoles are all excited with the same polarity. The vertical string contains all 24 of the vertical sextupoles. The horizontal string used to contain all 24 of the horizontal sextupoles, but now four of these, SHC8, SHF8, SHB4 and SHE4, have been taken out of the string so that they can be powered independently for resonant extraction. With these four sextupoles removed, there are four "holes" in the horizontal string, each hole having a partner located three superperiods away. This arrangement of horizontal sextupoles is depicted schematically in

Figure 9. Here the open red circles show the positions of the four holes; the filled circles show the positions of the remaining 20 horizontal sextupoles. The horizontal and vertical strings are powered by programmable monopolar power supplies that can deliver a maximum current of $300 \mathrm{~A}$ at a maximum of $90 \mathrm{~V}$.

The main windings on sextupoles SHC8, SHF8, SHB4 and SHE4 are connected together to form the $\mathrm{C}$ and $\mathrm{F}$ strings. These are the drive 
sextupole strings. The C string contains the SHC8 and SHE4 sextupoles; the F string contains SHF8 and SHB4. The two strings are depicted by the blue lines in Figure 9. Each string is powered with its own $\pm 350 \mathrm{~A}$ programmable power supply as indicated in Figure 10. Here the indicated polarities follow the conventions of Ref. [19]. The $Q_{H}=4+1 / 3$ resonance is excited by powering the two strings with opposite polarity.

The superperiod symmetry of the machine implies that the holes in the horizontal string will produce only even harmonics in azimuthal angle $\theta$ around the ring. Although this ensures that the $3 Q_{H}=13$ and

$Q_{H}+2 Q_{V}=13$ resonances (which are excited by harmonic 13) will not be excited, the $3 Q_{H}=14$ and $Q_{H}+2 Q_{V}=14$ resonances (which are excited by harmonic 14) can be excited. Note, however, that because the four holes are approximately equally spaced in betatron phase and in azimuth $\theta$, harmonics $2,6,10,14,18$, and so on, are suppressed to some extent. To ensure that the resonances are not excited during injection and acceleration, the currents in the four resonant extraction sextupoles will track the current in the horizontal string until extraction time.

The details of the excitation of the Horizontal, Vertical, C, and F strings for chromaticity adjustment and excitation of the $3 Q_{H}=13$ resonance are presented in Ref. [21].

\subsubsection{Resonance Correction Strings}

The auxiliary windings on the sextupoles are used for resonance correction as discussed in [22]. They are connected together to form eight strings, each string consisting of the auxiliary windings of 6 of the sextupoles connected in series as indicated below. The "+" and "-" signs indicate the polarity of each winding in the string, and "/2" indicates auxiliary windings with half as many turns as the others (i.e. with just one turn). We shall refer to the strings beginning with "SH" and "SV" as SH and SV strings.

$$
\begin{aligned}
& \text { SVSTR1: +SVA1 -SVA3 -SVC1/2 +SVC3/2 -SVE1/2 +SVE3/2 } \\
& \text { SHSTR1: +SHA2 -SHA4 -SHC2/2 +SHC4/2 -SHE2/2 +SHE4/2 } \\
& \text { SVSTR2: +SVA5 -SVA7 -SVC5/2+SVC7/2 -SVE5/2+SVE7/2 } \\
& \text { SHSTR2: +SHA6 -SHA8 -SHC6/2 +SHC8/2 -SHE6/2 +SHE8/2 } \\
& \text { SVSTR3: +SVD1 -SVD3 -SVF1/2 +SVF3/2 -SVB1/2 +SVB3/2 } \\
& \text { SHSTR3: +SHD2 -SHD4 -SHF2/2 +SHF4/2 -SHB2/2 +SHB4/2 }
\end{aligned}
$$


SVSTR4: +SVD5 -SVD7 -SVF5/2 +SVF7/2 -SVB5/2 +SVB7/2

SHSTR4: +SHD6 -SHD8 -SHF6/2 +SHF8/2 -SHB6/2 +SHB8/2

Each of the eight strings is connected to its own programmable power supply which is bipolar and can deliver a maximum current of 50 Amps at a maximum of 25 volts.

Note that in the SV strings the net EMF induced by the (changing) currents in the main windings of the magnets is zero. This is not always true for the SH strings. Each of these strings contains the auxiliary windings of one of the four sextupoles taken out of the horizontal chromaticity string. (These are the windings indicated by boldface type in the list above.) Thus, unless the currents in the main windings of SHE4, SHC8, SHB4, SHF8 follow the current in the horizontal chromaticity string, the net EMF induced in the $\mathrm{SH}$ strings will not be zero. If the $\mathrm{SH}$ string power supplies do not have enough voltage to cancel the induced $\mathrm{EMF}$, then the current required for resonance correction can not be maintained and the $3 Q_{H}=13, Q_{H}+2 Q_{V}=13,3 Q_{H}=14$, or $Q_{H}+2 Q_{V}=14$ resonances may be excited.

\section{Transport to Target Room}

The extraction channel of the D6 septum magnet feeds into the BAF transport line which is described in [3] and is shown schematically in Figure 11. The line consists of the indicated dipole (D1, D2) and quadrupole (Q1, Q2, .., Q8) magnets and brings the beam to the BAF target room. It is designed to provide a 2 to $20 \mathrm{~cm}$ diameter beam spot on the target. Two octupoles, one upstream of Q5 and the other upstream of Q6, can be adjusted to achieve a uniform rectangular distribution of beam on target as discussed in [3]. Note that there are fewer quadrupoles here than in the original design of the line, but the beam characteristics at the target are essentially the same [23].

\section{PPM Operation}

When the Booster Applications Facility becomes operational, we will want to inject and accelerate iron and proton beams in Booster on a PPM (Pulse-to-Pulse Modulation) basis. The iron ions will be delivered to BAF for biology experiments while protons will be delivered to the AGS and the 
experimental hall for HEP (high energy physics).

\section{$10.1 \quad$ Non-PPM Devices}

Most Booster devices that require different settings for proton and iron operation can be switched to the different settings on a PPM basis. The few exceptions are listed below.

\section{Booster Input Monitor and CBM (Circulating Beam}

Monitor). These require different setups for iron and proton operation. This is accomplished by "mode switching", but is not a PPM operation.

2. H-minus Injection Foil. Normally the foil is inserted into the Booster aperture for proton operation and retracted during heavy ion operation. The motorized device that does this can not move the foil in and out fast enough for PPM operation. (Even if it could, one would worry about the lifetime of such a device.) In Ref. [24] it is shown that in principle the injection foil can be left in its nominal position for H-minus injection without impinging on the available aperture for the injection and acceleration of heavy ions.

3. Booster RF systems. Some of the switching required here is not yet a PPM operation.

We note also that although switching the gains of the Booster Injection Current Transformer is a PPM operation, the signal from high-intensity protons on one Booster cycle can saturate the high-gain amplifier used to observe heavy-ions on a subsequent cycle.

\subsection{Joining the Iron and Proton Magnetic Cycles}

It is necessary that the field at the end of one magnetic cycle be equal the field at the beginning of the next. This can be accomplished by joining the proton cycle with the iron cycle as shown schematically in Figure 12. Here the field starts at the proton dwell field, but then must go down to the value required for iron injection. At injection, $B \rho$ is nominally $0.667705 \mathrm{Tm}$ for $\mathrm{Fe}^{20+}$ and $2.149636 \mathrm{Tm}$ for protons. The corresponding fields are 481.56 and 1550.3 gauss. Since the maximum voltage available to go down to the iron injection field is $1000 \mathrm{~V}$, Bdot is limited to $1.5 \mathrm{~T} / \mathrm{s}$ 
and the time required is at least $72 \mathrm{~ms}$. The actual magnetic cycle is shown in Figures 13 and 14. Here the flattop at $1000 \mathrm{MeV}$ per nucleon $(11.4 \mathrm{kG})$ is $400 \mathrm{~ms}$ long and the cycle length is $1700 \mathrm{~ms}$. The voltage is kept between \pm 1800 volts. The settings of the magnetic field used to generate the cycle are listed in Table 14. This magnetic cycle will fit in the $2.7667 \mathrm{~ms}$ long supercycle used for running the muon g-2 experiment. Here the available time for the iron cycle is the supercycle period minus the time required for the seven Booster proton magnetic cycles used for g-2 running. Since the proton cycles are $150 \mathrm{~ms}$ long, this amounts to $2767-1050=1717 \mathrm{~ms}$. The relative timing of the AGS and Booster magnetic cycles is shown schematically in Figure 15. 


\section{References}

[1] K.A. Brown, et al., "Design of a Resonant Extraction System for the AGS Booster", Proceedings of the 1999 Particle Accelerator Conference, New York, 1999, pp. 1270-1272.

[2] K.A. Brown, et al., "Resonant Extraction Parameters for the AGS Booster", Proceedings of the 2001 Particle Accelerator Conference, Chicago, 2001, pp. 1409-1411.

[3] N. Tsoupas, et al., "The Booster Applications Facility (BAF) Beam Transport Line of the BNL AGS Booster", Proceedings of the 1999 Particle Accelerator Conference, New York, 1999, pp. 1267-1269.

[4] David R. Lide (Editor-in-Chief), Handbook of Chemistry and Physics, 80th Edition, 1999-2000, CRC Press LLC, 1999, pp. 1-10 through $1-12$.

M.A. Zucker and R.A. Dragoset (2000). Elemental Data Index (Version 1.1), [Online]. Available: http://physics.nist.gov/EDI [2000, September 6]. National Institute of Standards and Technology, Gaithersburg, MD.

[5] D.E. Groom, et al., The European Physical Journal C 15, 73 (2000)

[6] C.J. Gardner, "Booster Inflector Specifications", Booster Tech. Note No. 159, February 28, 1990.

[7] K.L. Zeno, "Overview of the YEAR $2000 \mathrm{Au}^{32+}$ Booster Run", C-A/AP/Note 26, October, 2000.

[8] C.J. Gardner, et al., "Status and Recent Performance of the Accelerators that Serve as Gold Injector for RHIC", Proceedings of the 2001 Particle Accelerator Conference, Chicago, 2001, pp. $3184-3186$.

[9] C.J. Gardner, et al., "Injection of Gold Ions in the AGS Booster with Linear Coupling", Proceedings of the 1999 Particle Accelerator Conference, New York, 1999, pp. 1276-1278.

[10] S.Y. Zhang and L.A. Ahrens, "Gold Beam Losses at the AGS Booster Injection", Proceedings of the 1999 Particle Accelerator Conference, New York, 1999, pp. 3294-3296. 
[11] C.J. Gardner, "RF Capture and Acceleration of Gold Ions in Booster-II", C-A/AP/Note 28, October, 2000.

[12] R. Thern, "Booster Dipole Production Measurements", Booster Tech. Note 190, March 13, 1991.

[13] E. Bleser, "Booster Short Quadrupole Production Measurements", Booster Tech. Note No. 174, September 12, 1990.

[14] E. Bleser, "Booster Long Quadrupole Production Measurements", Booster Tech. Note No. 176, September 13, 1990.

[15] A. Luccio, "Algorithm and Charts to Calculate and Modify Tunes and Chromaticity in teh AGS Booster, Proton Case", Booster Tech. Note No. 179, October 17, 1990.

[16] W. van Asselt, "Booster Tune Control", AGS/AD/Operations Note No. 40, February 4, 1993.

[17] C.J. Gardner and W. van Asselt, "Booster Tune Control Limits at High Field", Booster Tech. Note 220, February 10, 1993.

[18] K.A. Brown, W. van Asselt, and W. Meng, "A High Precision Model of Booster Tune Control", C-A/AP Note 69, February, 2002.

[19] E. Bleser, "Booster Polarity Standards", Booster Technical Note 180, October 30, 1990.

[20] E. Bleser, "Booster Sextupole Production Measurements", Booster Tech. Note No. 182, 13 March 1991.

[21] C.J. Gardner, "Programming the New Sextupole Strings in Booster", forthcoming C-A/AP Note.

[22] C.J. Gardner, "The Booster Stopband Correction System-1997", AGS/AD/Tech. Note 465, July 22, 1997.

[23] P. Pile, private communication.

[24] C.J. Gardner, "Multi-turn Injection of Heavy-Ions in Booster with the H-Minus Injection Foil Inserted", Collider-Accelerator Dept. Note C-A/AP/Note 64, September, 2001. 


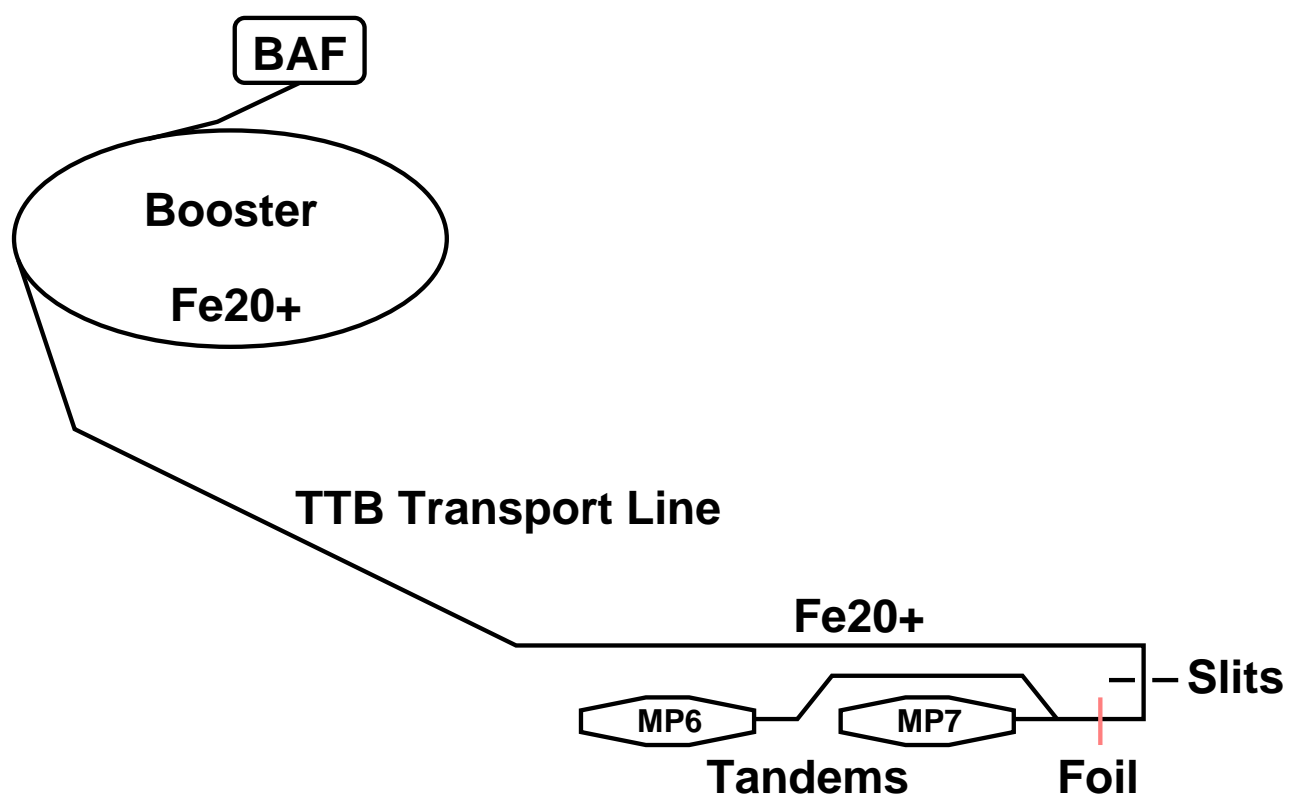

Figure 1: Layout of Accelerators for BAF

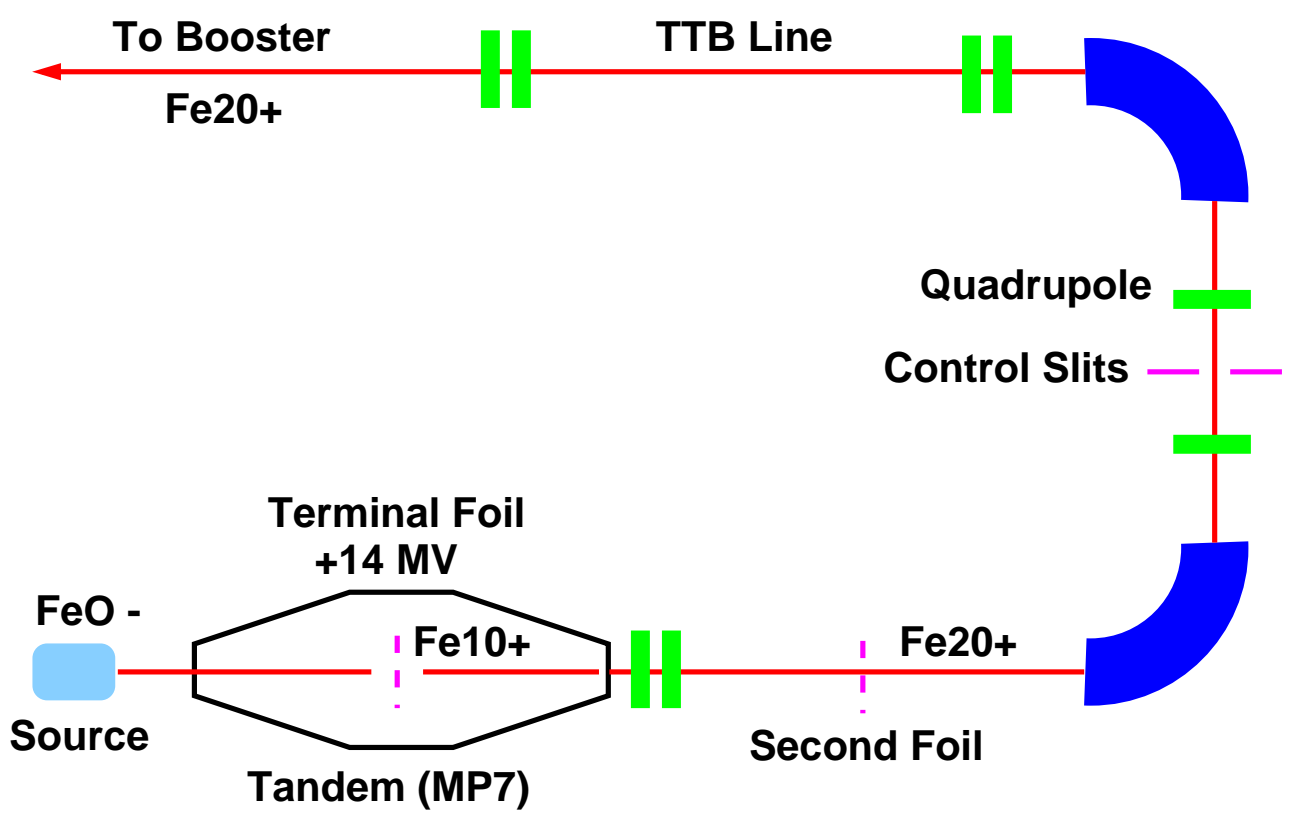

Figure 2: Detail of MP7 Tandem and Beginning of TTB Line. 


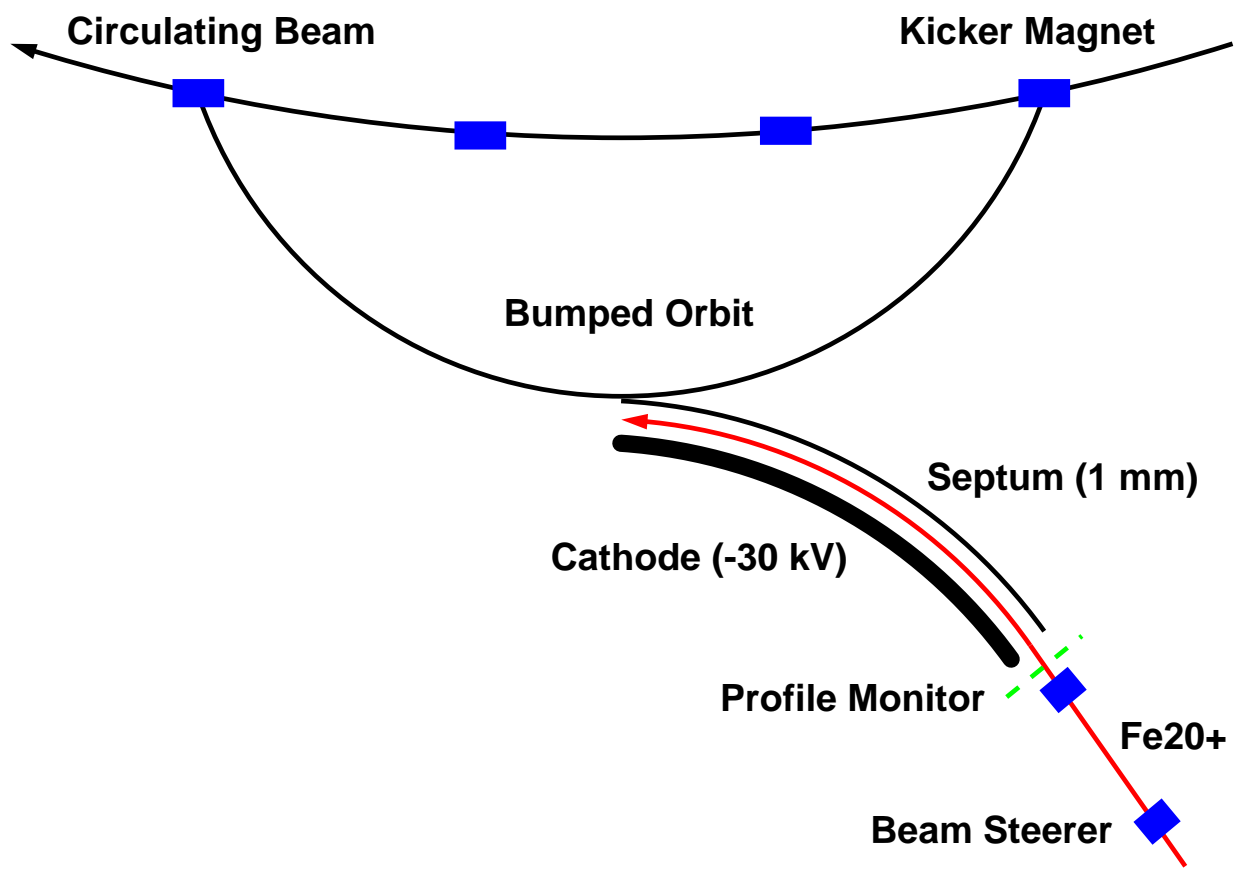

Figure 3: Components for the Injection of Iron in Booster. The electrostatic inflector (consisting of the cathode and septum as indicated) is located in the C3 straight section. The kickers are located in the C1, C3, C7, and D1 straights. The closed orbit bump produced by the kickers initially places the orbit near the septum at the exit of the inflector. As beam is injected and begins to circulate, the bump must be collapsed at a rate sufficient to keep the circulating beam from hitting the septum. 


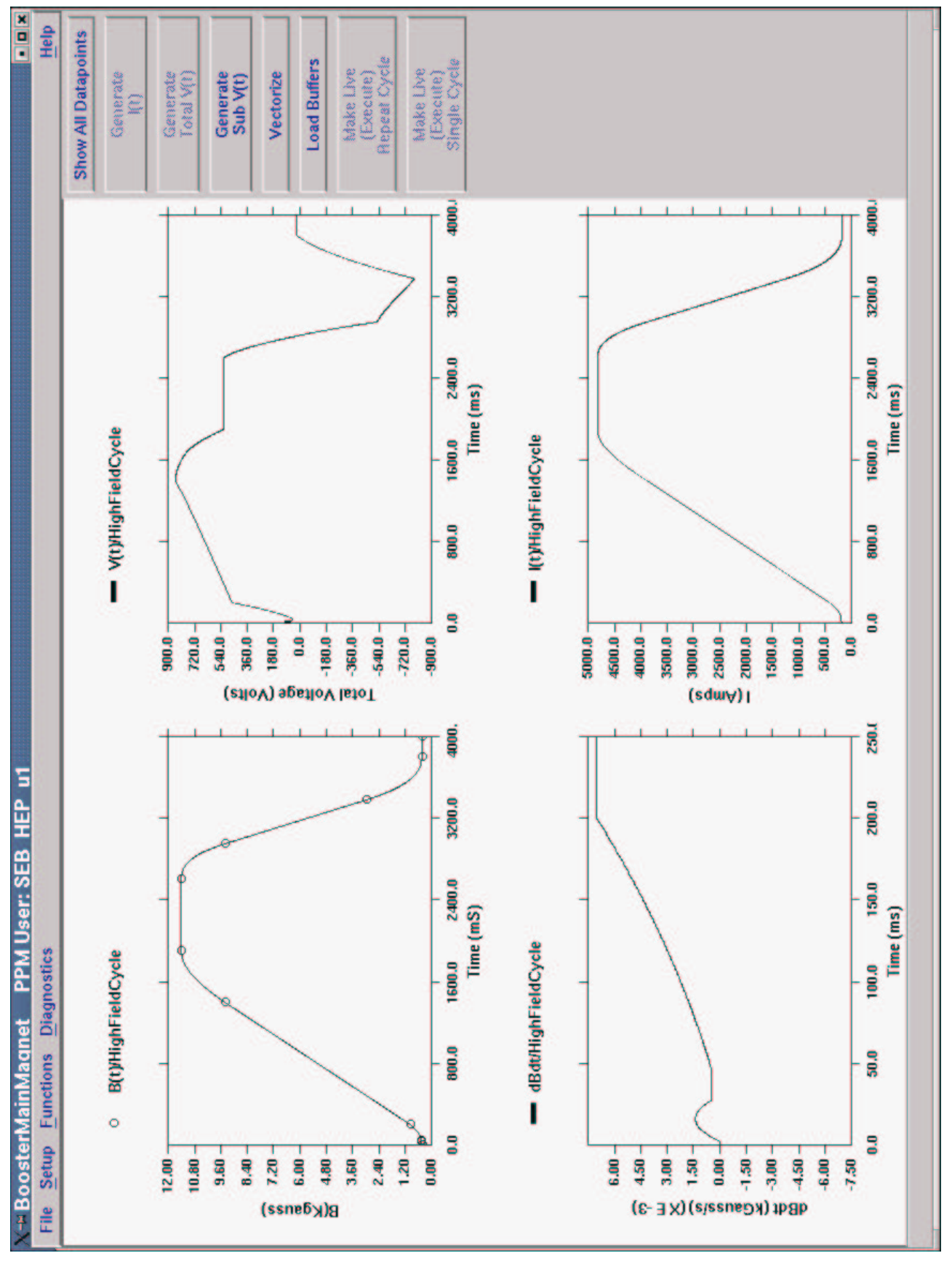

Figure 4: Main Magnet Functions using One High-Current Module 
Table 12: Magnetic Cycle Parameters for Figure 4

\begin{tabular}{|c|c|c|c|}
\hline$t(\mathrm{~ms})$ & $B(\mathrm{kG})$ & Interpolation & $m(\mathrm{kG} / \mathrm{ms})$ \\
\hline 0 & 0.450 & Linear & 0 \\
\hline 2 & 0.450 & Linear & 0 \\
\hline 27 & 0.475 & Cubic & 0.0005 \\
\hline 47 & 0.485 & Linear & 0.0005 \\
\hline 200 & 1.000 & Cubic & 0.007 \\
\hline 1400 & 9.400 & Linear & 0.007 \\
\hline 1900 & 11.400 & Cubic & 0 \\
\hline 2600 & 11.400 & Linear & 0 \\
\hline 2950 & 9.400 & Cubic & -0.015 \\
\hline 3376.67 & 3.000 & Linear & -0.015 \\
\hline 3800 & 0.450 & Cubic & 0 \\
\hline 4000 & 0.450 & Linear & 0 \\
\hline
\end{tabular}

Table 13: Magnetic Cycle Parameters for Figure 5

\begin{tabular}{|c|c|c|c|}
\hline$t(\mathrm{~ms})$ & $B(\mathrm{kG})$ & Interpolation & $m(\mathrm{kG} / \mathrm{ms})$ \\
\hline 0 & 0.450 & Linear & 0 \\
\hline 2 & 0.450 & Linear & 0 \\
\hline 27 & 0.475 & Cubic & 0.0005 \\
\hline 47 & 0.485 & Linear & 0.0005 \\
\hline 150 & 2.000 & Cubic & 0.020 \\
\hline 550 & 10.000 & Linear & 0.020 \\
\hline 700 & 11.400 & Cubic & 0 \\
\hline 1400 & 11.400 & Linear & 0 \\
\hline 1500 & 10.000 & Cubic & -0.030 \\
\hline 1766.67 & 2.000 & Linear & -0.030 \\
\hline 1870 & 0.450 & Cubic & 0 \\
\hline 2000 & 0.450 & Linear & 0 \\
\hline
\end{tabular}




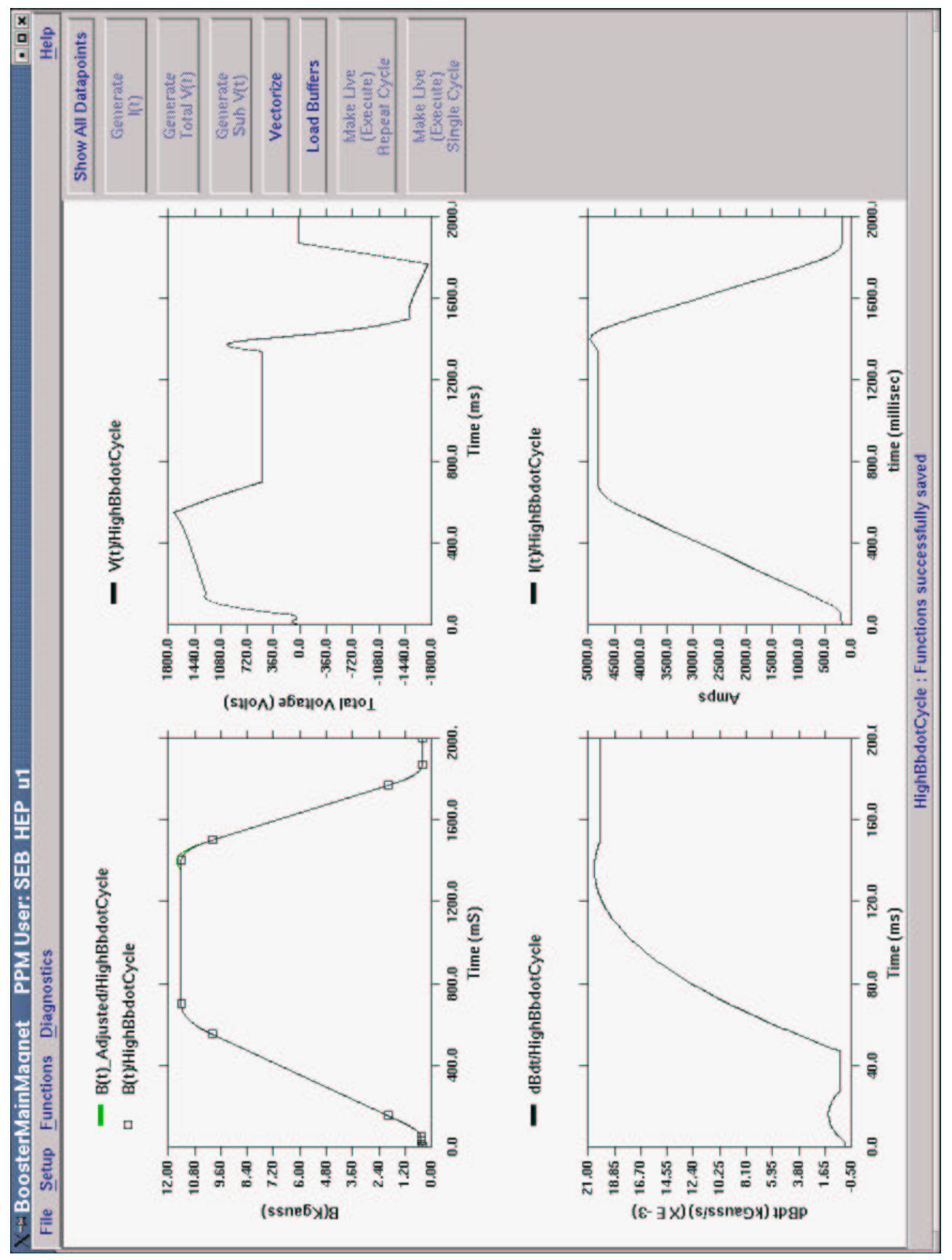

Figure 5: Main Magnet Functions using Two High-Current Modules 


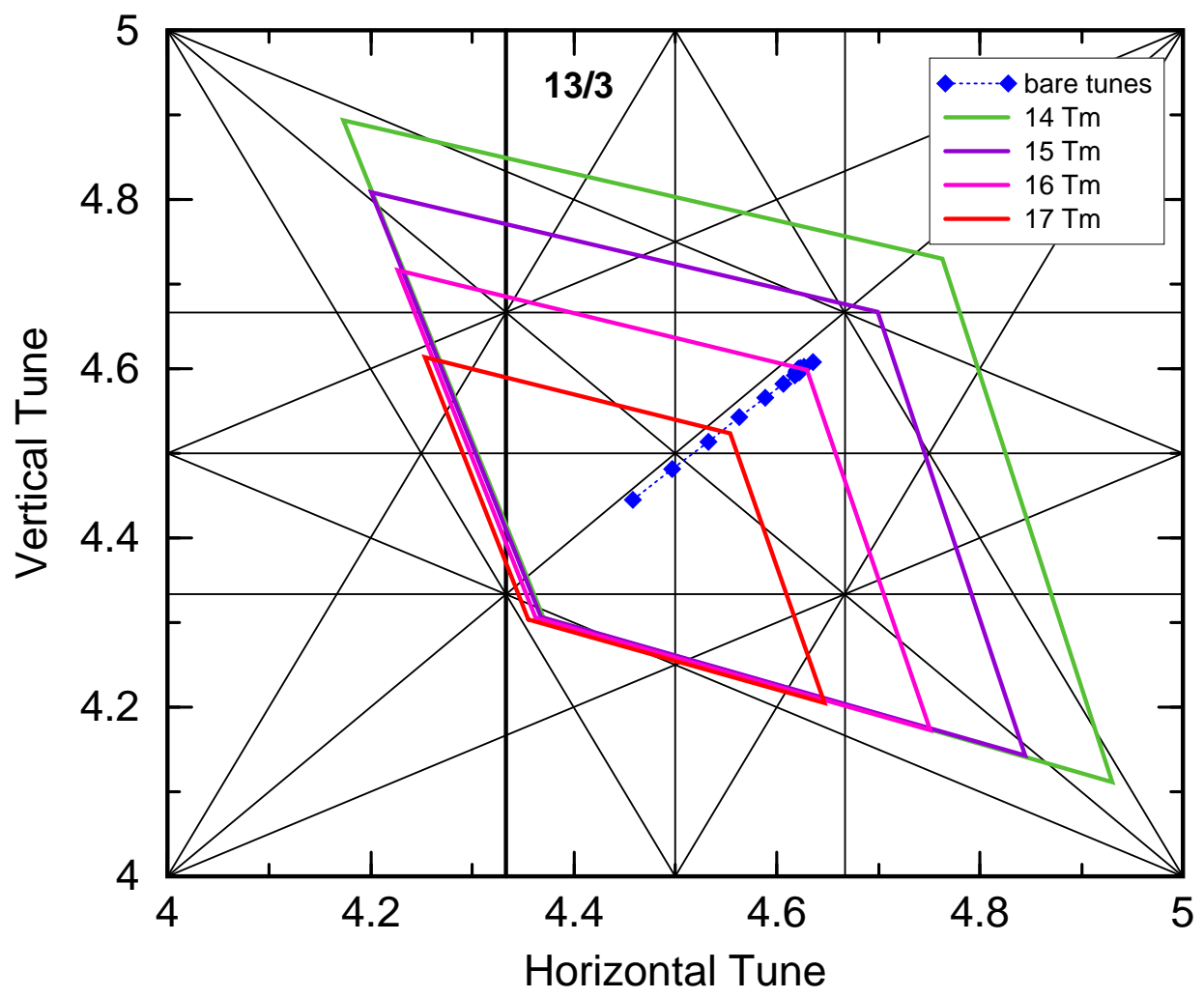

Figure 6: Booster Tune Control at High Field 


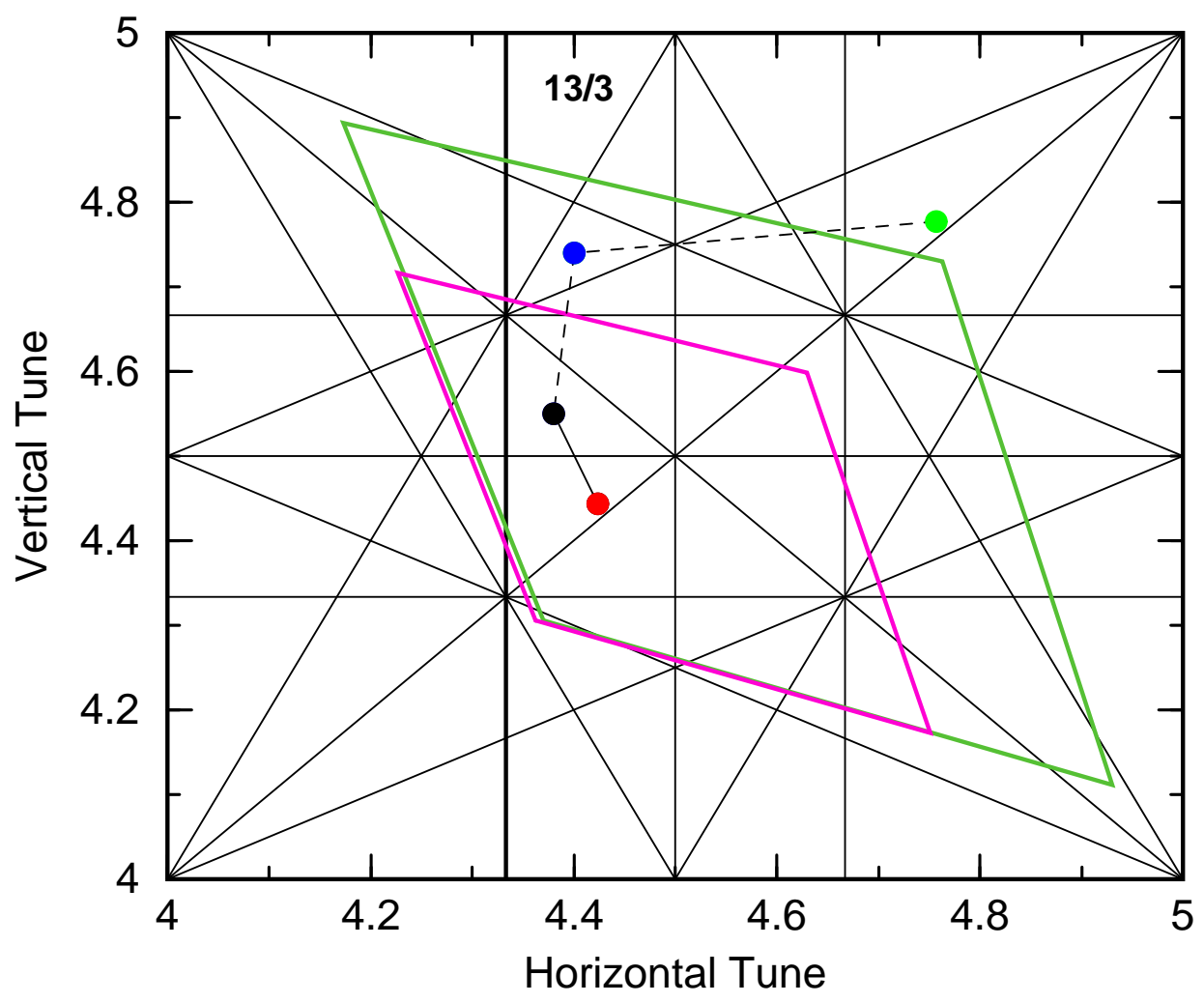

Figure 7: Tune Manipulation at Injection. Injection occurs with the tunes at the green point. Shortly after injection, the tunes are moved to the blue point and then to the black point. Here the tunes sit until extraction. During extraction, the horizontal tune is moved to the left across the $Q_{H}=4+1 / 3$ resonance. An alternative scheme has the tunes sitting at the red point during injection. Shortly after, the tunes are again moved to the black point where they sit until extraction. 

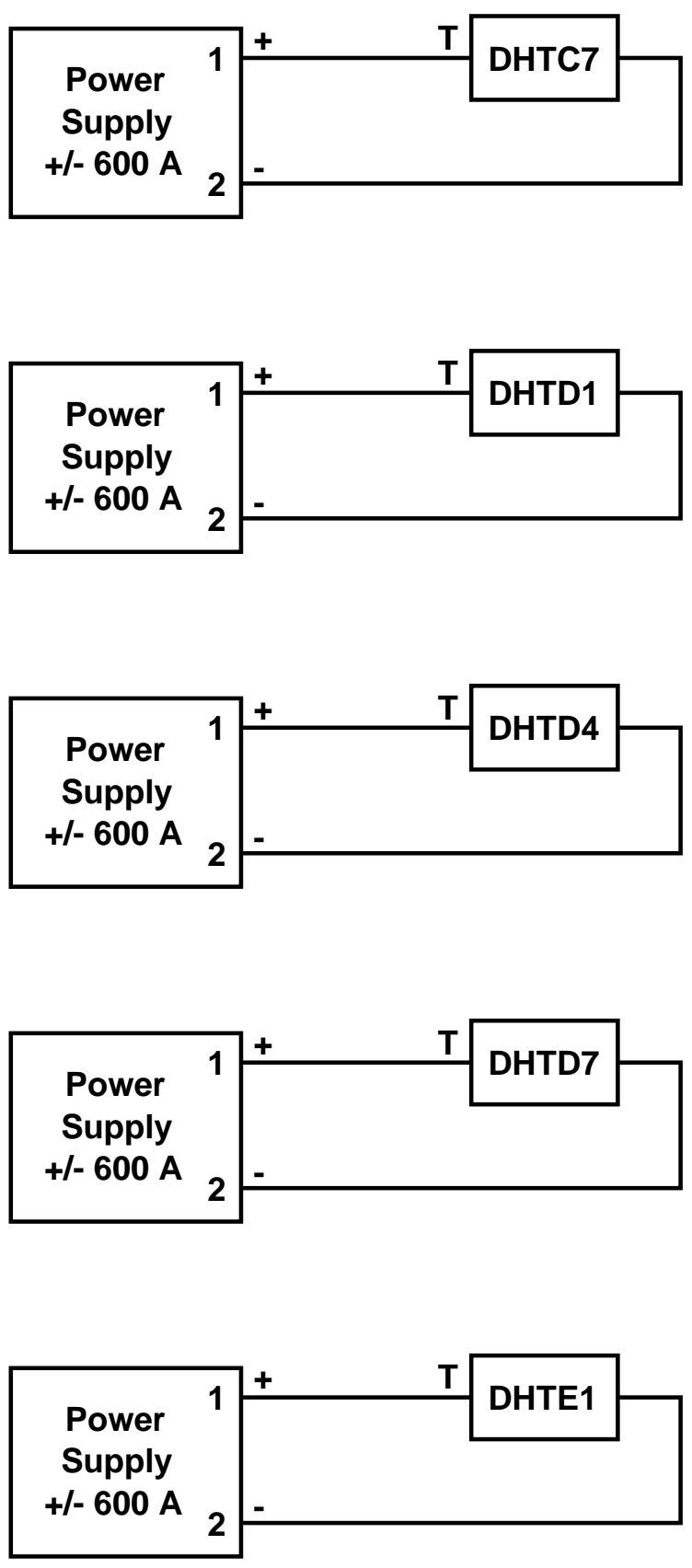

Figure 8: Wiring Diagram for BAF Extraction Bump Magnets. (Here the prefix DHT stands for Dipole Horizontal Trim.) The polarity convention is that a positive reference signal at the power supply input produces positive current out of the terminal labeled "1". A positive current into the "T" lead of the magnet puts the magnet in "A" polarity as per Ref. [19]. 


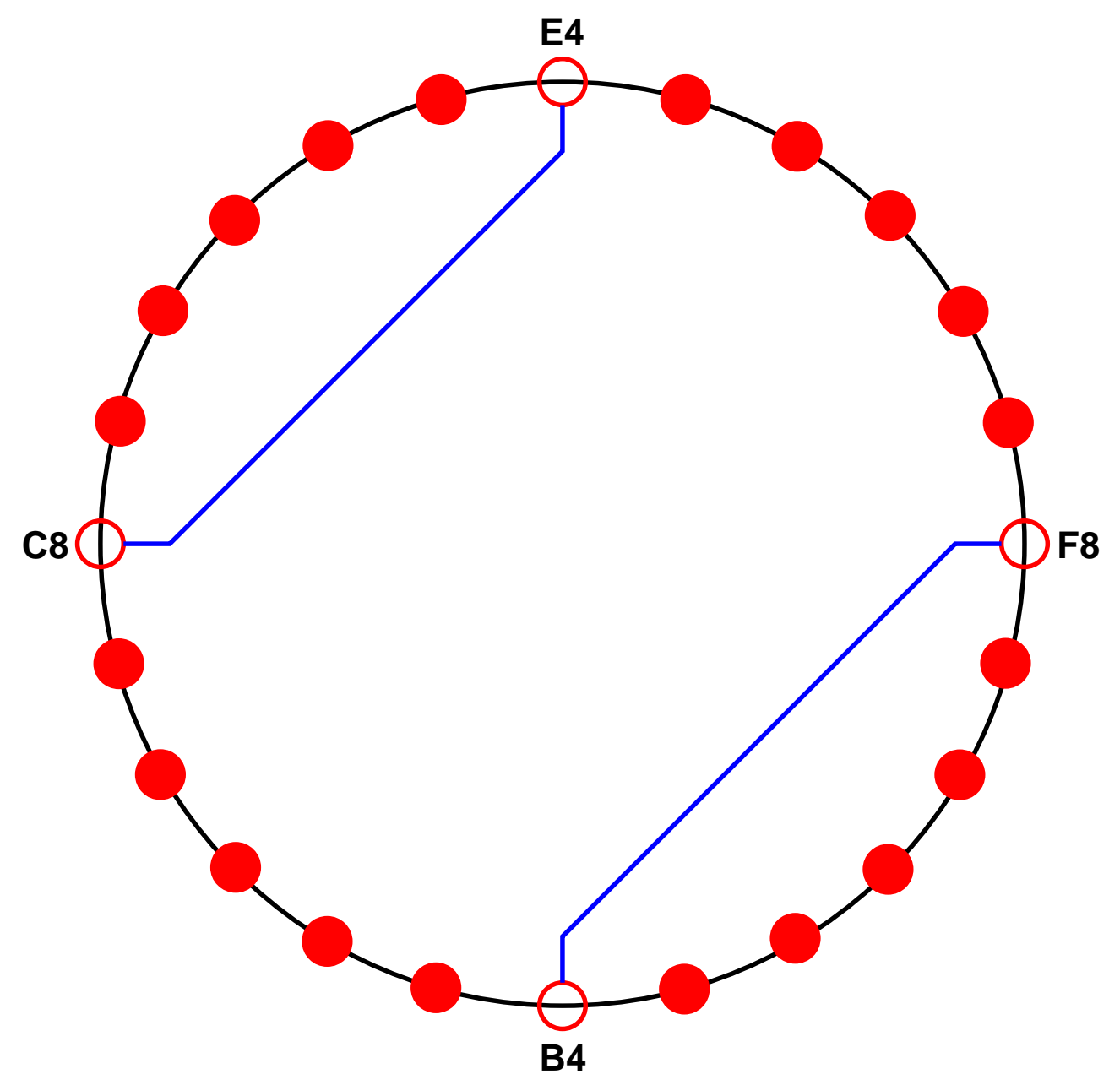

Figure 9: Horizontal Sextupoles in the Booster Ring. The open circles show the positions of the four drive sextupoles. The filled circles show the positions of the remaining 20 horizontal sextupoles. 

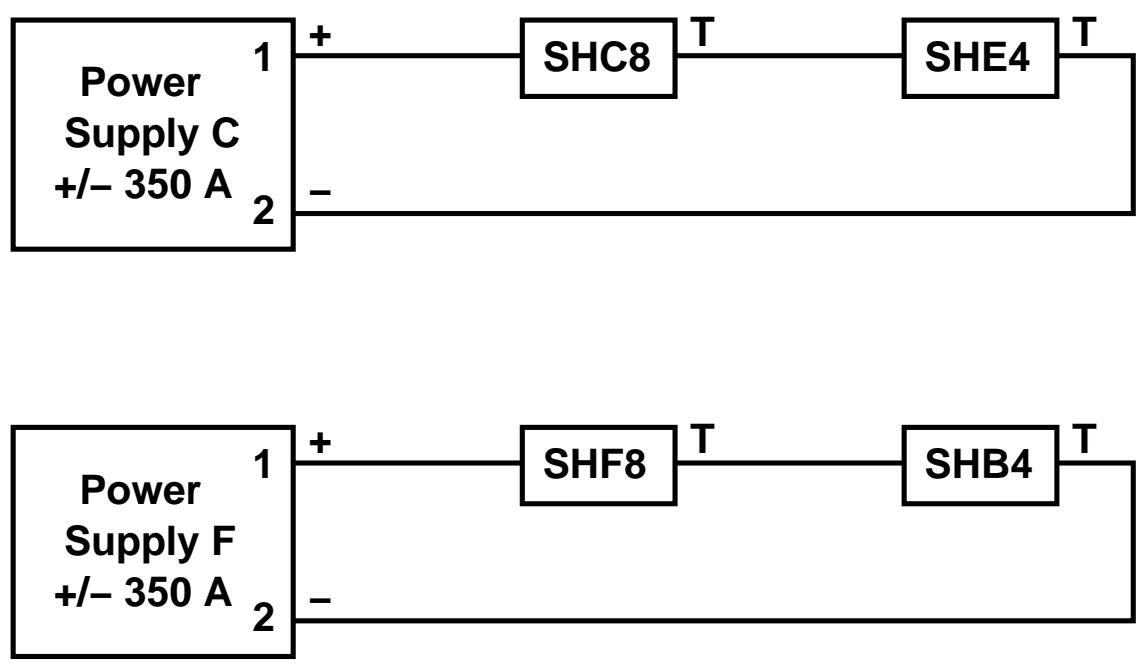

Figure 10: Wiring Diagram for the C and F Sextupole Strings. (Here the prefix SH stands for Sextupole Horizontal.) The polarity convention is that a positive reference signal at the power supply input produces positive current out of the terminal labeled "1". A positive current into the "T" lead of the magnet puts the magnet in "A" polarity as per Ref. [19]. Note that in the control system, power supplies C and F are called "b-sxr1-ps" and "b-sxr2ps" respectively. 


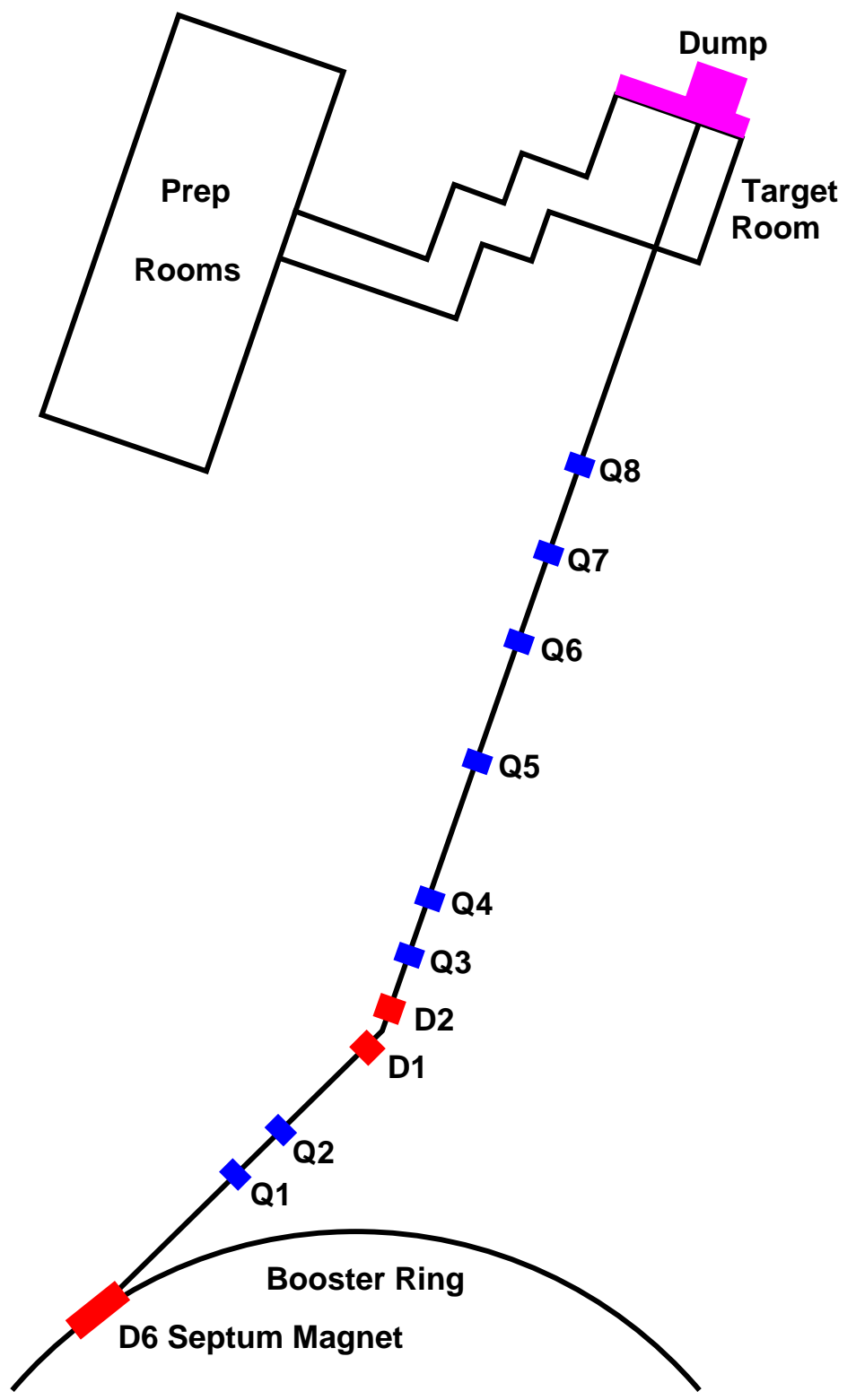

Figure 11: BAF Transport Line 


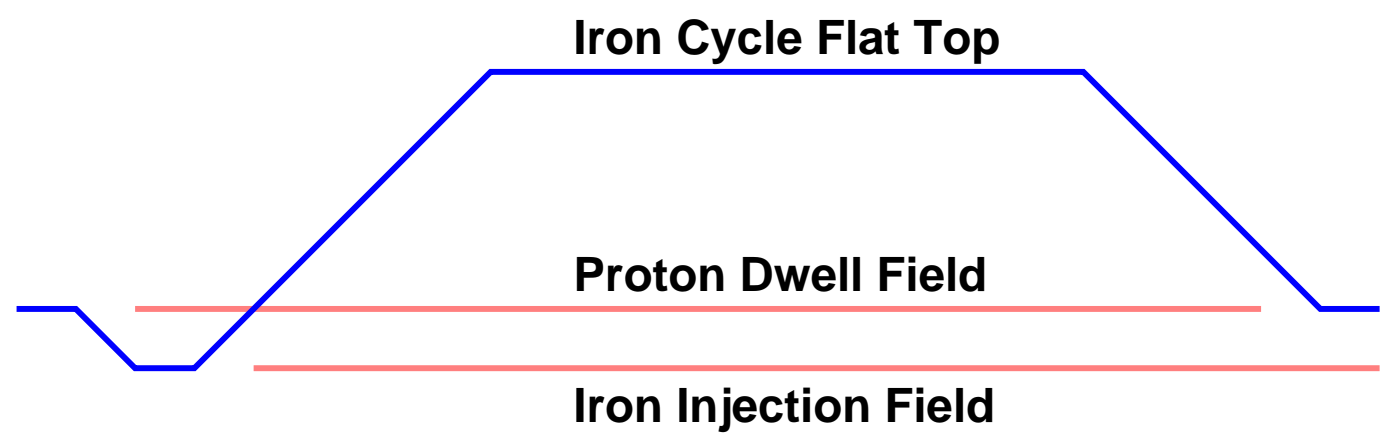

Figure 12: Iron Magnetic Cycle for PPM with HEP

Table 14: Magnetic Cycle Parameters for Figure 13

\begin{tabular}{|c|c|c|c|}
\hline$t(\mathrm{~ms})$ & $B(\mathrm{kG})$ & Interpolation & $m(\mathrm{kG} / \mathrm{ms})$ \\
\hline 0 & 1.380 & Linear & 0 \\
\hline 2 & 1.380 & Linear & 0 \\
\hline 100 & 0.450 & Cubic & 0 \\
\hline 102 & 0.450 & Linear & 0 \\
\hline 127 & 0.475 & Cubic & 0.0005 \\
\hline 147 & 0.485 & Linear & 0.0005 \\
\hline 250 & 2.000 & Cubic & 0.020 \\
\hline 650 & 10.000 & Linear & 0.020 \\
\hline 800 & 11.400 & Cubic & 0 \\
\hline 1200 & 11.400 & Linear & 0 \\
\hline 1300 & 10.000 & Cubic & -0.030 \\
\hline 1566.67 & 2.000 & Linear & -0.030 \\
\hline 1620 & 1.380 & Cubic & 0 \\
\hline 1700 & 1.380 & Linear & 0 \\
\hline
\end{tabular}




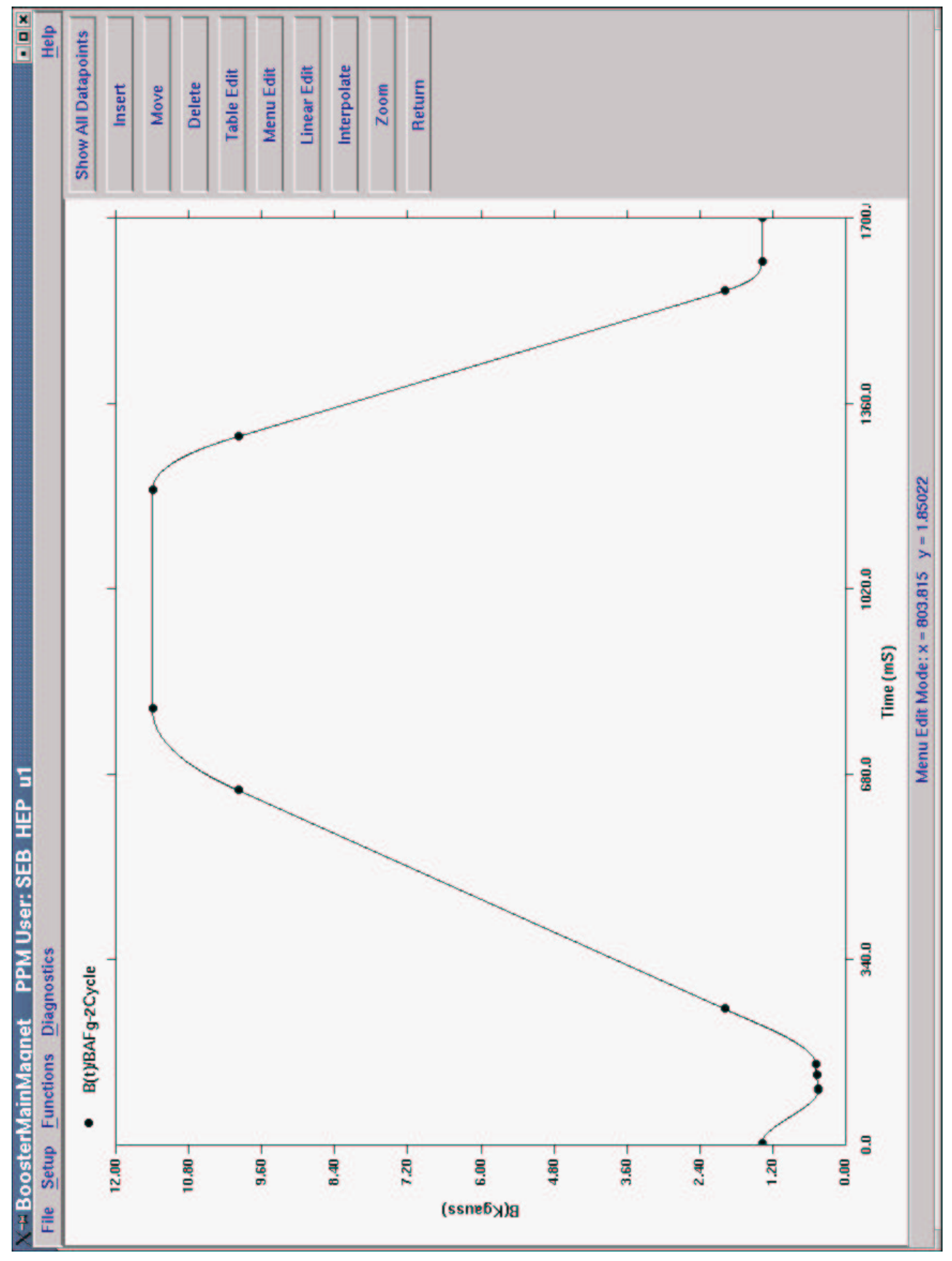

Figure 13: Iron Magnetic Cycle for PPM with HEP 


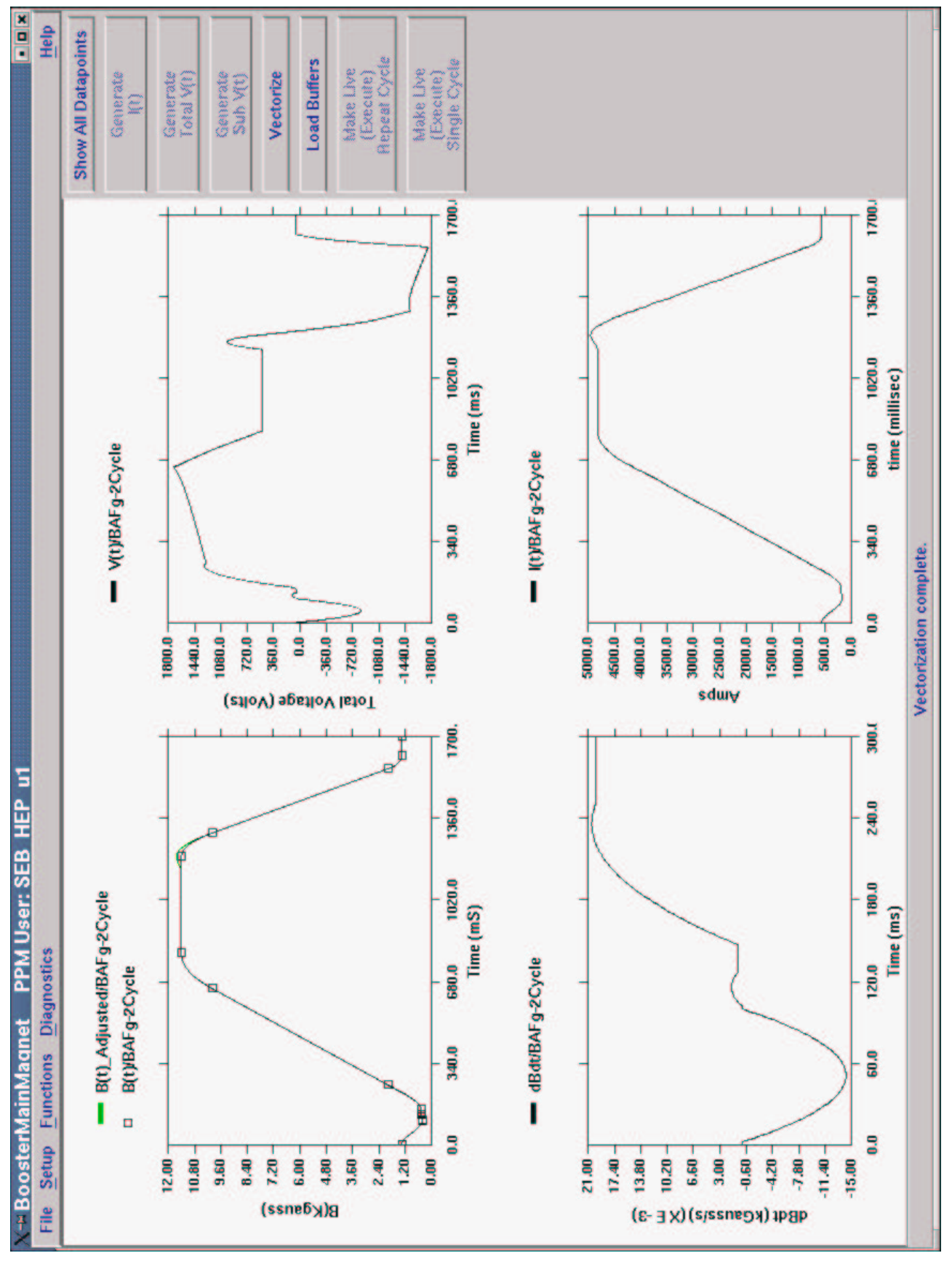

Figure 14: Iron Magnetic Cycle Functions for PPM with HEP 


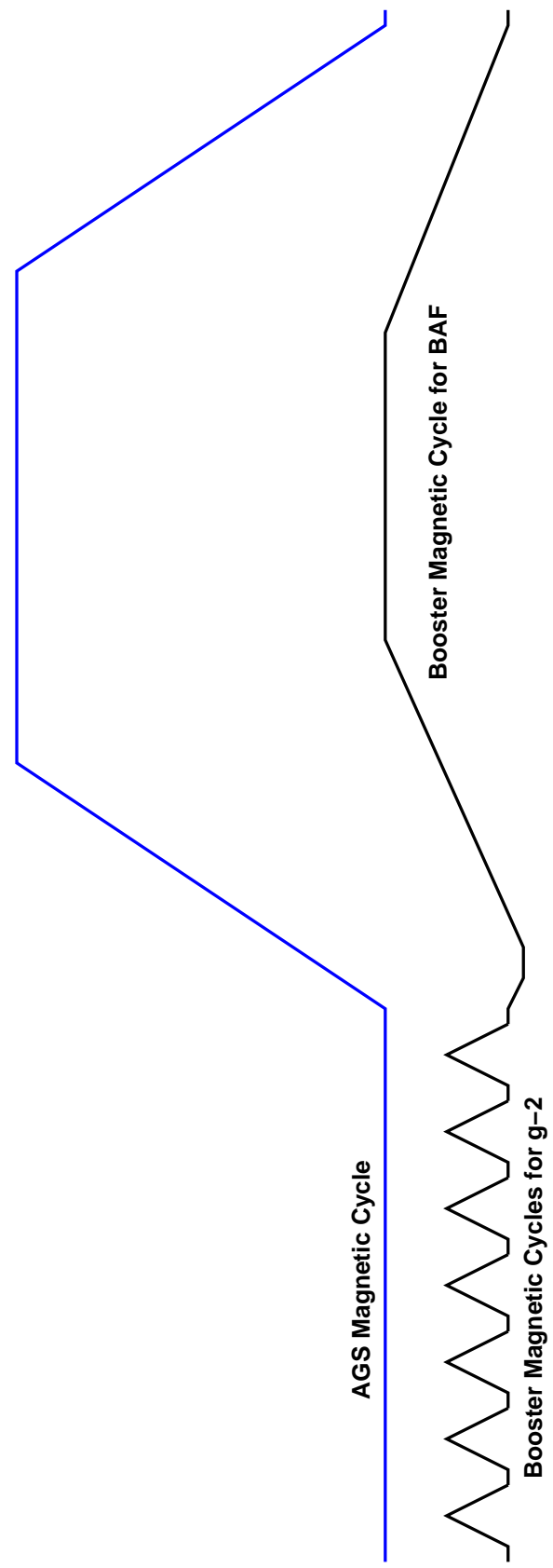

Figure 15: Magnetic Cycles for running BAF and g-2 simultaneously. 